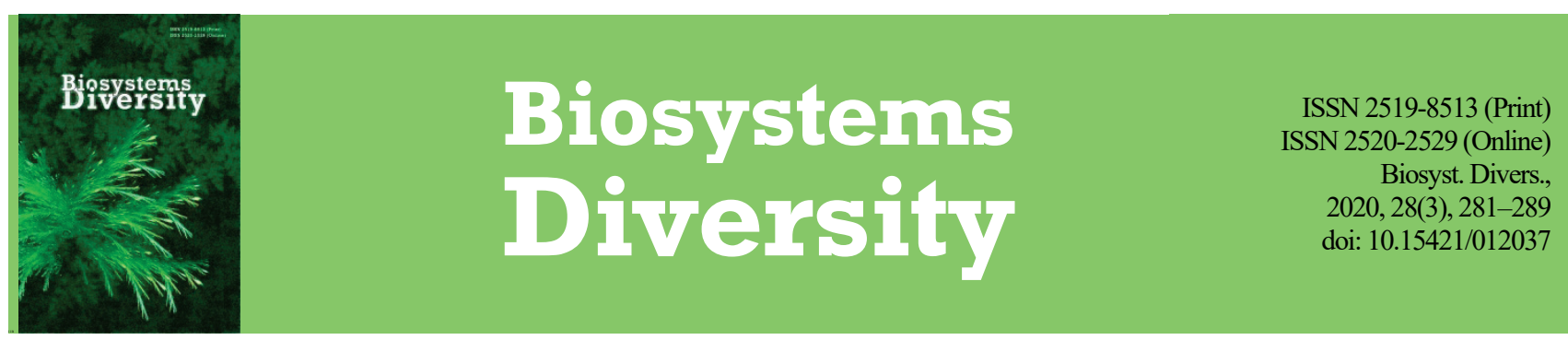

\title{
Antibacterial and fungicidal activities of ethanol extracts of 38 species of plants
}

\author{
V. V. Zazharskyi*, P. O. Davydenko*, O. M. Kulishenko*, I. V. Borovik*, N. M. Zazharska*, V. V. Brygadyrenko**** \\ *Dnipro State Agrarian and Economic University, Dnipro, Ukraine \\ **Oles Honchar Dnipro National University, Dnipro, Ukraine
}

Article info

Received 19.06.2020

Received in revised form 02.08 .2020

Accepted 04.08.2020

Dnipro State Agrarian and Economic University, Sergiy Efremov st., 25 , Dnipro, 49000, Ukraine. Tel.: +38-056-713-51-74. E-mail:

zazharskiyv@gmail.com

Oles Honchar

Dnipro National University, Gagarin av., 72, Dnipro, 49010, Ukraine. Tel.: +38-050-93-90-788 E-mail:brigad@ua.fm

\author{
Zazharskyi, V. V., Davydenko, P. O., Kulishenko, O. M., Borovik, I. V., Zazharska, N. M., \& Brygadyrenko, V. V. (2020). Antibacteri- \\ al and fungicidal activities of ethanol extracts of 38 species of plants. Biosystems Diversity, 28(3), 281-289. doi:10.15421/012037
}

Galenic preparations are broadly used against microorganisms pathogenic to humans, thought their poteintial in this aspect is not studied completely. In our in vitro experiment we studied the influence of alcohol tinctures from 38 species of plants on 15 species of bacteria and one species of fungus. Zones of growth inhibition of colonies measuring over $8 \mathrm{~mm}$ were observed during the use of ethanol extracts of Maclura pomifera against eight species of microorganisms (Escherichia coli, Proteus mirabilis, Serratia marcescens, Yersinia enterocolitica, Salmonella typhimurium, Rhodococcus equi, Campylobacter jejuni and Corynebacterium xerosis), Ginkgo biloba - against eight species (Enterococcus faecalis, S. marcescens, Y. enterocolitica, Klebsiella pneumoniae, Listeria innocua, L. monocytogenes, $P$. aeruginosa and C. jejuni), Genista tinctoria - against seven species (E. coli, Enterobacter aerogenes, Proteus mirabilis, K. pneumoniae, S. typhimurium, P. aeruginosa and Rh. equi), Phellodendron amurense-against seven species (E. faecalis, S. marcescens, S. typhimurium, Rh. equi, C. jejuni, C. xerosis and Candida albicans), Berberis vulgaris - against seven species ( $P$. mirabilis, S. marcescens, K. pneumoniae, S. typhimurium, C. jejuni, P. aeruginosa and C. xerosis), Vitex negundoagainst six species (E. faecalis, E. coli, P. mirabilis, K. pneumoniae, S. typhimurium and Rh. equi), Koelreuteria paniculata-against six species (E. faecalis, P. mirabilis, $S$. marcescens, S. typhimurium, C. jejuni and E. coli), Magnolia kobus - against six species (E. faecalis, E. coli, P. mirabilis, S. marcescens, S. typhimurium, C. jejuni and C. xerosis), Liriodendron tulipifera - against six species (K. pneumoniae, Listeria innocua, P. aeruginosa, C. jejuni, Rh. equi and C. albicans), Clematis flammula - against six species (E. faecalis, P. mirabilis, L. monocytogenes, P. aeruginosa, C. jejuni and C. xerosis), Wisteria sinensis - against five species (E. coli, S. typhimurium, L. monocytogenes, Rh. equi and C. albicans), Chimonanthus praecox - against five species (E. faecalis, S. marcescens, L. monocytogenes, C. jejuni and Rh. equi), Colchicum autumnale - against five species (S. marcescens, K. pneumoniae, L. ivanovi, L. monocytogenes and $P$. aeruginosa). As a result of the study, these plants were found to be the most promising for further study of in vivo antibacterial activity. In the search of antibacterial and antifungal activities, the following plants were observed to be less promising: Ailanthus altissima, Aristolochia manshuriensis, Artemisia absinthium, Callicarpa bodinieri, Campsis radicans, Catalpa duclouxii, Celastrus scandens, Dictamnus alba, Eucommia ulmoides, Geranium sanguineum, Laburnum anagyroides, Nepeta racemosa, Parthenocissus tricuspidata, Polygonatum multiflorum, Prunus dulcis, P. laurocerasus, Ptelea trifoliata, Pteridium aquilinum, Quercus castaneifolia, Q. petraea iberica, Salvia officinalis, Securigera varia, Styphnolobium japonicum, Tamarix elongata and Vitex agnus-castus.

Keywords: growth inhibition zone; bacterial colonies; multi-resistant strain; candidosis

\section{Introduction}

During recent years there have been reports from all over the globe about resistance to medicine of disease-causing bacteria of human and animals. Emergence of resistance to antibiotics in bacteria is a global problem (Lopes et al., 2018; Zhang et al., 2019). Antibiotic-resistance is now one of the most serious threats to the health of people (Steinberg et al., 2017; Tumen et al., 2018; Khan et al., 2019). Our civilization is approaching the period when antibiotics will be unable to control the courses of common infections, and small traumas could once more lead to people dying (Islam et al., 2019). Antibiotics have allowed humans to live longer and be healthier. Resistance to preparations for treatment of a common intestinal bacterium Klebsiella pneumonia (carbapenems) occurs more and more frequently. K. pneumonia can cause various nosocomial infections (pneumonia, infections of blood, infections among newborns, etc.). Resistance to fluoroquinolones used for treatment of urinary tract infections caused by $E$. coli has also become widely distributed. In the 1980s, when these preparations were first used, resistance to them was practically absent. Currently, in many countries this treatment is ineffective for over $50 \%$ of patients. Cases of no effect on gonorrhea treatment with reserve antibiotics - cephalosporins of the third generation - have been confirmed in many countries of the EU, Australia, Canada, South
Africa and Japan. Salević et al. (2019) report that probability of death of humans infected with MRSA (methicillin-resistant Staphylococcus aureus) is $64 \%$ higher compared with people with medically-non-resistant form of this infection. Resistance to antibiotics among microorganisms also leads to increase in costs for medical services due to the longer period of stay in hospitals. Therefore, there is a necessity of developing alternative antimicrobial preparations for treatment of infectious diseases. Rates of development of new antimicrobial preparations should exceed the rates of development of resistance among microorganisms to currently in-use antibiotics.

In this article we continue to study antibacterial preparations in plant extracts due to spread of antibiotic poly-resistant bacterial strains which are hard to treat (Zazharskyi et al., 2019; Palchykov et al., 2020). Plants produce various secondary metabolites with different biological activity. For galenic preparations of some species of plants we have already found anti-parasitic and antimicrobial activities (Boyko \& Brygadyrenko, 2016a; Palchykov et al., 2019; Zazharskyi et al., 2019b).

The objective of this article was determining antibacterial effects of 38 ethanol extracts on 16 species of microorganisms. Up to now, these species of plants have remained poorly studied with respect to antimicrobial activity and can have significant potential in contemporary human and veterinary medicine. 


\section{Material and methods}

Leaves and shoots of 38 species of plants were collected in the territory of the Botanical Garden of Oles Honchar Dnipo National University (Khromykh et al., 2018; Boyko \& Brygadyrenko, 2019), dried at room temperature, fragmented, weighed and kept in $70 \%$ ethyl alcohol for 10 days, and then filtered. We took 10 grams of dry fragmented plants per $100 \mathrm{~g}$ of $70 \%$ ethyl alcohol. Then, $0.1 \mathrm{~mL}$ of this filtered alcohol extract was transferred onto one paper disk of $6 \mathrm{~mm}$ diameter. The disks were dried in sterile conditions in the temperature of $10^{\circ} \mathrm{C}$ in a microbiological safety cabinet HR1200-IIA2-D (China).

Table 1

Taxonomic composition of 16 species of microorganisms we studied

\begin{tabular}{|c|c|c|}
\hline Phylum, Division & Family & $\begin{array}{l}\text { Species, strains } \\
\end{array}$ \\
\hline \multirow{5}{*}{ Proteobacteria } & Yersiniaceae & $\begin{array}{l}\text { Serratia marcescens ATCC } 8100 \\
\text { Yersinia enterocolitica ATCC } 9610\end{array}$ \\
\hline & Enterobacteriaceae & $\begin{array}{l}\text { Enterobacter aegorenes ATCC } 10006 \\
\text { Escherichia coli } 055 \\
\text { Klebsiella pneumoniae ATCC } 13883 \\
\text { Salmonella typhimurium ATCC } 14028\end{array}$ \\
\hline & Morganellaceae & Proteus mirabilis ATCC 14153 \\
\hline & Pseudomonadaceae & Pseudomonas aeruginosa ATCC 2353 \\
\hline & Campylobacteraceae & Campylobacterjejuni ATCC 11322 \\
\hline \multirow[b]{2}{*}{ Firmicutes } & Enterococcaceae & Enterococcus faecalis ATCC 19433 \\
\hline & Listeriaceae & $\begin{array}{l}\text { Listeria ivanovii } \\
\text { L. innocua ATCC } 33090 \\
\text { L. monocytogenes ATCC } 19112\end{array}$ \\
\hline \multirow{2}{*}{ Actinobacteria } & Nocardiaceae & Rhodococcus equi ATCC 6939 \\
\hline & Corynebacteriaceae & Corynebacterium xerosis 1911 \\
\hline Ascomycota & Saccharomycetaceae & Candida albicans ATCC 2091 \\
\hline
\end{tabular}

Table 2

Parts of the 38 species of plants we used to prepare ethanol extracts and the most important data on their antibacterial activities

\begin{tabular}{|c|c|c|c|}
\hline Family & Species & Used part of plant & Most important literature sources about medical properties of plant \\
\hline Aristolochiaceae & Aristolochia manshuriensis Kom. & leaves & Kavitha \& Nelson, 2016 \\
\hline Asparagaceae & Polygonatum multiflorum (L.) All. & leaves & Bährle-Rapp, 2007 \\
\hline Asteraceae & Artemisia absinthium L. & leaves & Obistioiu \& Chiurciu, 2014, Al-Ghamdi, 2020 \\
\hline Berberidaceae & Berberis vulgaris L. & leaves & Özgen \& Geçer, 2012; Anzabi, 2018 \\
\hline Bignoniaceae & Campsis radicans (L.) Seem. & leaves & Islam \& Haque, 2019 \\
\hline Bignoniaceae & Catalpa duclouxii Dode & leaves & Zhang et al., 2018 \\
\hline Calycanthaceae & Chimonanthus praecox (L.) Link & leaves & Gui \& Qin, 2014 \\
\hline Celastraceae & Celastrus scandens $\mathrm{L}$. & leaves & Kumar \& Sharma, 2018 \\
\hline Colchicaceae & Colchicum autumnale $\mathrm{L}$. & leaves & Adami \& Naderi, 2015 \\
\hline Dennstaedtiaceae & Pteridium aquilinum (L.) Kuhn & leaves & Kardong \& Saikia, 2013 \\
\hline Eucommiaceae & Eucommia ulmoides Oliv. & bark & Liu \& Han, 2007; Zhang \& An, 2019 \\
\hline Fabaceae & Genista tinctoria $\mathrm{L}$. & shoots and leaves & Geraldes \& Costa, 2019 \\
\hline Fabaceae & Laburnum anagyroides Medik. & leaves & Rivers, 2016 \\
\hline Fabaceae & Securigera varia (L.) Lassen & leaves & Behbahani et al., 2013 \\
\hline Fabaceae & Styphnolobium japonicum (L.) Schott & shoots and leaves & Lim, 2013 \\
\hline Fabaceae & Wisteria sinensis (Sims) Sweet & leaves & Compton, 2015 \\
\hline Fagaceae & Quercus castaneifolia C. A. Mey. & shoots and leaves & Bahador \& Baserisalehi, 2011 \\
\hline Fagaceae & Q. petraea iberica (Steven ex M. Bieb.) Krassiln. & shoots and leaves & Tumen \& Sekeroglu, 2018 \\
\hline Geraniaceae & Geranium sanguineum $\mathrm{L}$. & shoots and leaves & Bigos \& Sienkiewicz, 2012; Wafa \& Ouarda, 2017 \\
\hline Ginkgoaceae & Ginkgo biloba L. & leaves & Xie \& Johnson, 2003 \\
\hline Lamiaceae & Callicarpa bodinieri H. Lév. & leaves & $\mathrm{Ma} \& \mathrm{Su}, 2015$ \\
\hline Lamiaceae & Nepeta racemosa Lam. & leaves & Saxena \& Mathela, 1996; Mathela \& Joshi, 2008 \\
\hline Lamiaceae & Salvia officinalis L. & leaves & Salević \& Lagaron, 2019; Wali \& Alam, 2019 \\
\hline Lamiaceae & Vitex agnus-castus $\mathrm{L}$. & leaves & Habbab \& Aboul-Enein, 2016 \\
\hline Lamiaceae & $V$. negundo L. & shoots and leaves & $\begin{array}{l}\text { Prashith \& Raghavendra, 2014; Sharma \& Suri, 2016; } \\
\text { Triveni \& Gaddad, } 2016\end{array}$ \\
\hline Magnoliaceae & Liriodendron tulipifera $\mathrm{L}$. & leaves & Hufford \& Robertson, 1975 \\
\hline Magnoliaceae & Magnolia kobus DC. & leaves & $\mathrm{Hu} \& \mathrm{Ge}, 2011$ \\
\hline Moraceae & Maclura pomifera (Raf.) C. K. Schneid. & leaves & Allen, 1985; Dharmaratne \& Nanayakkara, 2013 \\
\hline Ranunculaceae & Clematis flammula $\mathrm{L}$. & leaves & Khan \& Omoloso, 2001; Buzzini \& Pieroni, 2003 \\
\hline Rosaceae & Prunus dulcis (Mill.) D. A. Webb & fruits and leaves & Thebo, 2014 \\
\hline Rosaceae & P. laurocerasus L. & leaves & Akpulat \& Enginoğlu, 2019 \\
\hline Rutaceae & Dictammus alba L. & leaves & Lei \& Liao, 2007 \\
\hline Rutaceae & Phellodendron amurense Rupr. & shoots and leaves & Wang \& Zhang, 2009; Han \& Meng, 2015 \\
\hline Rutaceae & Ptelea trifoliata $\mathrm{L}$. & bark & Steinberg \& Setzer, 2017 \\
\hline Sapindaceae & Koelreuteria paniculata Laxm. & leaves & Mostafa \& Ross, 2015 \\
\hline Simaroubaceae & Ailanthus altissima (Mill.) Swingle & leaves & Albouchi \& Hosni, 2013 \\
\hline Tamaricaceae & Tamarix elongata Ledeb. & leaves & Saïdana \& Helal, 2008 \\
\hline Vitaceae & Parthenocissus tricuspidata (Siebold \& Zucc.) Planch. & leaves & Park et al., 2008 \\
\hline
\end{tabular}
microorganisms we prepared a weighed amount according to the standard of opacity of bacterial suspension equaling 0.5 units of density according to McFarland (McF) $1.5 \times 10^{8} \mathrm{CFU}$ (colony-forming units), which we determined using a densitometer (Densimeter II, Table 1).

The obtained weighed amount was transferred into Muller-Hinton agar (Himedia) with subsequent cultivation in a TCO-80/1 thermostat for $24 \mathrm{~h}$ at the temperature of $37^{\circ} \mathrm{C}$. On top of the inoculations, we put disks $(n=8)$ saturated with corresponding ethanol tinctures of 38 species of plants (Table 2). 
As a positive control we used disks with $15 \mu \mathrm{g}$ of azithromycin - broadspectrum macrolide (Valle et al., 2015). Disck with $15.0 \mu \mathrm{g}$ amphotericinin were also used as a second control against Candida albicans. Twenty four hours later the growth of the culture was measured using a zone scale for reading the sizes of growth inhibition zones of microorganisms (Antibiotic Zone Scale-C, model PW297, India) and software TpsDig2 (2016, F. James Rohlf). The data in the tables are presented as $\mathrm{x} \pm \mathrm{SD}$ (standart deviation).

\section{Results}

Growth of separate strains of microorganisms of Enterococcaceae, Enterobacteriaceae, Morganellaceae and Yersiniaceae families was arrested by ethanol extracts of the species of plants we studied (Table 3, 4). We observed inhibition of growth of E. faecalis by Ginkgo biloba $(18.4 \mathrm{~mm}$, hereinafter the average radius of growth inhibition zone is given in $\mathrm{mm}$ ). Slightly lower than Ginkgo biloba, but still high antibacterial effects were exhibited by Pteridium aquilinum (14.5), Polygonatum multiflorum (12.4), Clematis flammula (10.6), Magnolia kobus (10.4), Prunus laurocerasus (10.2), Vitex negundo (10.2). Moderate inhibition of growth of the colonies of $E$. faecalis was seen under the influence of Dictammus albus (9.8), Koelreuteria paniculata (8.4), Chimonanthus praecox (8.2), Celastrus scandens (6.7) and Callicarpa bodinieri (6.2). Bacteria of $E$. faecalis were resistant to the influence of alcohol extracts of the rest of the species of plants we studied.

Against $E$. aerogenes bacteria of Enterobacteriaceae, only one ethanol extract demonstrated competition to azithromycin (17.8) - Genista tinctoria (12.6). Moderate inhibition of growth of colonies of $E$. aerogenes was exerted by extracts from Catalpa duclouxii (5.7), Liriodendron tulipifera (4.3), Pteridium aquilinum (4.3), Quercus castaneifolia (3.8), Aristolochia manshuriensis (3.7), Parthenocissus tricuspidata (3.6), Salvia officinalis (3.5). At the same time, one should note the complete absence of the reaction of this strain (complete absence of arrest of growth of bacterial colonies) to ethyl extracts of such plants as Vitex negundo, $V$. agnus-castus, Styphnolobium japonicum, Artemisia absinthium, Maclura pomifera, Koelrenteria paniculata, Phellodendron amurense, Prunus dulcis, Eucommia ulmoides, Wisteria sinensis, Laburnum anagyroides, Securigera varia, Celastrus scandens, Quercus petraea, Ptelea trifoliata.

High susceptibility of $E$. coli was seen to 10 ethanol extracts: Prunus laurocerasus (12.7), Polygonatum multiflorum (11.8), Koelreuteria paniculata (11.5), Wisteria sinensis (10.9), Quercus castaneifolia (10.7), Prunus dulcis (10.5), Styphnolobium japonicum (10.5), Vitex negundo (10.3); moderate susceptibility - to eight species of plants: Machura pomifera (9.8), Celastrus scandens (9.5), Artemisia absinthium (7.7), Laburnum anagyroides (7.4), Quercus petraea iberica (6.8), Catalpa duclouxii (6.7), Colchicum autumnale (6.5) and Liriodendron tulipifera (6.4). The poly-resistant strain of E. coli was insensitive to Genista tinctoria, Eucommia ulmoides, Geranium sanguineum, Nepeta racemosa, Tamarix elongata and Ptelea trifoliata. We also determined that azithromycin in the control was ineffective against $P$. mirabilis, whereas high antibacterial effects were exerted by some alcohol extracts of plants: Parthenocissus tricuspidata (13.6), Celastrus scandens (12.7), Vitex negundo (12.6), Maclura pomifera (12.4), Magnolia kobus (12.4), V. agnus-castus (11.2), Catalpa duclouxii (10.7), Ailanthus altissima (10.4) and Quercus castaneifolia (10.4).

Table 3

Antibacterial effect of ethanol extracts of plants on Enterococcus faecalis, Enterobacter aerogenes, Escherichia coli and Proteus mirabilis $(\mathrm{x} \pm \mathrm{SD}, \mathrm{n}=8)$

\begin{tabular}{|c|c|c|c|c|c|c|c|c|c|}
\hline \multirow{2}{*}{ Family } & \multirow{2}{*}{ Species } & \multicolumn{2}{|c|}{ E. faecalis } & \multicolumn{2}{|c|}{ E. aerogenes } & \multicolumn{2}{|c|}{ E. coli } & \multicolumn{2}{|c|}{ P. mirabilis } \\
\hline & & test & control ${ }^{*}$ & test & control ${ }^{*}$ & test & control" & test & control $^{*}$ \\
\hline Aristolochiaceae & Aristolochia manshuriensis Kom. & $0 \pm 0$ & $25.7 \pm 3.21$ & $3.7 \pm 0.33$ & $17.3 \pm 1.79$ & $4.8 \pm 0.34$ & $15.4 \pm 1.42$ & $5.6 \pm 0.44$ & $0 \pm 0$ \\
\hline Asparagaceae & Polygonatum multiflorum (L.) All. & $12.4 \pm 1.34$ & $26.7 \pm 4.34$ & $2.4 \pm 0.12$ & $19.9 \pm 1.76$ & $11.8 \pm 1.42$ & $17.9 \pm 1.74$ & $2.8 \pm 0.22$ & $0 \pm 0$ \\
\hline Asteraceae & Artemisia absinthium L. & $2.2 \pm 0.36$ & $25.6 \pm 3.12$ & $0 \pm 0$ & $16.9 \pm 1.88$ & $7.7 \pm 0.67$ & $15.7 \pm 1.59$ & $2.3 \pm 0.23$ & $0 \pm 0$ \\
\hline Berberidaceae & Berberis vulgaris L. & $0 \pm 0$ & $26.7 \pm 3.21$ & $1.6 \pm 0.14$ & $17.7 \pm 1.66$ & $1.7 \pm 0.17$ & $15.8 \pm 1.61$ & $8.8 \pm 0.87$ & $0 \pm 0$ \\
\hline Bignoniaceae & Campsis radicans (L.) Seem. & $0 \pm 0$ & $26.5 \pm 2.98$ & $2.6 \pm 0.39$ & $18.6 \pm 1.77$ & $4.6 \pm 0.44$ & $13.8 \pm 1.73$ & $2.4 \pm 0.26$ & $0 \pm 0$ \\
\hline Bignoniaceae & Catalpa duclouxii Dode & $2.1 \pm 0.34$ & $24.3 \pm 2.98$ & $5.7 \pm 0.53$ & $19.5 \pm 1.88$ & $6.7 \pm 0.54$ & $16.3 \pm 1.61$ & $10.7 \pm 0.87$ & $0 \pm 0$ \\
\hline Calycanthaceae & Chimonanthus praecox (L.) Link & $8.2 \pm 1.31$ & $24.7 \pm 3.38$ & $2.7 \pm 0.26$ & $17.4 \pm 1.78$ & $3.7 \pm 0.25$ & $17.4 \pm 1.52$ & $2.2 \pm 0.24$ & $0 \pm 0$ \\
\hline Celastraceae & Celastrus scandens $\mathrm{L}$. & $6.7 \pm 1.22$ & $27.6 \pm 2.43$ & $0 \pm 0$ & $16.4 \pm 1.96$ & $9.5 \pm 0.98$ & $16.1 \pm 2.58$ & $12.7 \pm 1.12$ & $0 \pm 0$ \\
\hline Colchicaceae & Colchicum autumnale L. & $0 \pm 0$ & $25.7 \pm 2.78$ & $3.2 \pm 0.34$ & $18.8 \pm 1.63$ & $6.5 \pm 0.41$ & $14.7 \pm 1.59$ & $2.7 \pm 0.23$ & $0 \pm 0$ \\
\hline Dennstaedtiaceae & Pteridium aquilinum (L.) Kuhn & $14.5 \pm 1.67$ & $24.8 \pm 2.67$ & $4.3 \pm 0.32$ & $16.4 \pm 1.87$ & $2.7 \pm 0.32$ & $15.4 \pm 1.48$ & $0 \pm 0$ & $0 \pm 0$ \\
\hline Eucommiaceae & Eucommia ulmoides Oliv. & $0 \pm 0$ & $23.7 \pm 1.98$ & $0 \pm 0$ & $18.8 \pm 1.64$ & $0 \pm 0$ & $13.7 \pm 1.51$ & $0 \pm 0$ & $0 \pm 0$ \\
\hline Fabaceae & Genista tinctoria L. & $0 \pm 0$ & $24.6 \pm 1.73$ & $12.6 \pm 1.24$ & $17.8 \pm 1.51$ & $0 \pm 0$ & $17.2 \pm 1.77$ & $9.3 \pm 0.76$ & $0 \pm 0$ \\
\hline Fabaceae & Laburnum anagyroides Medik. & $0 \pm 0$ & $27.5 \pm 4.67$ & $0 \pm 0$ & $18.2 \pm 1.57$ & $7.4 \pm 0.56$ & $13.4 \pm 1.47$ & $0 \pm 0$ & $0 \pm 0$ \\
\hline Fabaceae & Securigera varia (L.) Lassen & $0 \pm 0$ & $26.3 \pm 4.33$ & $0 \pm 0$ & $18.8 \pm 1.73$ & $2.6 \pm 0.21$ & $15.9 \pm 1.58$ & $2.9 \pm 0.31$ & $0 \pm 0$ \\
\hline Fabaceae & Styphnolobium japonicum (L.) Schott & $0 \pm 0$ & $24.6 \pm 1.74$ & $0 \pm 0$ & $18.6 \pm 1.62$ & $10.5 \pm 1.45$ & $15.9 \pm 1.68$ & $2.7 \pm 0.14$ & $0 \pm 0$ \\
\hline Fabaceae & Wisteria sinensis (Sims) Sweet & $0 \pm 0$ & $25.8 \pm 3.24$ & $0 \pm 0$ & $17.7 \pm 1.67$ & $10.9 \pm 1.45$ & $15.4 \pm 1.49$ & $0 \pm 0$ & $0 \pm 0$ \\
\hline Fagaceae & Quercus castaneifolia C. A. Mey. & $2.5 \pm 0.45$ & $24.8 \pm 2.16$ & $3.8 \pm 0.45$ & $17.5 \pm 1.45$ & $10.7 \pm 1.32$ & $14.3 \pm 1.48$ & $10.4 \pm 0.84$ & $0 \pm 0$ \\
\hline Fagaceae & $\begin{array}{l}\text { Quercus petraea iberica } \\
\text { (Steven ex M. Bieb.) Krassiln. }\end{array}$ & $0 \pm 0$ & $26.5 \pm 1.98$ & $0 \pm 0$ & $18.5 \pm 1.61$ & $6.8 \pm 0.45$ & $14.6 \pm 1.59$ & $1.5 \pm 0.13$ & $0 \pm 0$ \\
\hline Geraniaceae & Geranium sanguineum $\mathrm{L}$. & $0 \pm 0$ & $24.2 \pm 1.78$ & $2.3 \pm 0.34$ & $16.7 \pm 1.45$ & $0 \pm 0$ & $14.7 \pm 1.63$ & $2.6 \pm 0.17$ & $0 \pm 0$ \\
\hline Ginkgoaceae & Ginkgo biloba L. & $18.4 \pm 1.98$ & $24.8 \pm 2.16$ & $2.7 \pm 0.15$ & $16.3 \pm 1.87$ & $4.5 \pm 0.32$ & $15.3 \pm 1.34$ & $1.6 \pm 0.17$ & $0 \pm 0$ \\
\hline Lamiaceae & Callicarpa bodinieri H. Lév. & $6.2 \pm 0.76$ & $25.7 \pm 2.98$ & $2.2 \pm 0.14$ & $15.5 \pm 1.65$ & $2.7 \pm 0.33$ & $15.5 \pm 1.52$ & $2.4 \pm 0.15$ & $0 \pm 0$ \\
\hline Lamiaceae & Nepeta racemosa Lam. & $0 \pm 0$ & $25.8 \pm 3.45$ & $1.2 \pm 0.11$ & $16.5 \pm 1.86$ & $0 \pm 0$ & $15.5 \pm 1.63$ & $1.6 \pm 0.36$ & $0 \pm 0$ \\
\hline Lamiaceae & Salvia officinalis L. & $0 \pm 0$ & $26.7 \pm 3.98$ & $3.5 \pm 0.42$ & $18.8 \pm 1.77$ & $5.4 \pm 0.45$ & $16.7 \pm 1.64$ & $3.8 \pm 0.31$ & $0 \pm 0$ \\
\hline Lamiaceae & Vitex agnus-castus L. & $4.1 \pm 0.47$ & $25.4 \pm 2.68$ & $0 \pm 0$ & $19.3 \pm 1.31$ & $2.8 \pm 0.17$ & $15.4 \pm 1.44$ & $11.2 \pm 0.94$ & $0 \pm 0$ \\
\hline Lamiaceae & Vitex negundo $\mathrm{L}$. & $10.2 \pm 0.94$ & $25.2 \pm 2.16$ & $0 \pm 0$ & $17.8 \pm 1.65$ & $10.3 \pm 1.24$ & $15.4 \pm 1.64$ & $12.6 \pm 0.89$ & $0 \pm 0$ \\
\hline Magnoliaceae & Liriodendron tulipifera $\mathrm{L}$. & $0 \pm 0$ & $24.7 \pm 2.70$ & $4.3 \pm 0.47$ & $16.7 \pm 1.86$ & $6.4 \pm 0.62$ & $15.5 \pm 1.58$ & $6.5 \pm 0.75$ & $0 \pm 0$ \\
\hline Magnoliaceae & Magnolia kobus DC. & $10.4 \pm 1.67$ & $25.3 \pm 1.89$ & $1.2 \pm 0.14$ & $16.5 \pm 1.93$ & $3.5 \pm 0.23$ & $14.2 \pm 1.59$ & $12.4 \pm 1.32$ & $0 \pm 0$ \\
\hline Moraceae & $\begin{array}{l}\text { Machura pomifera (Raf.) } \\
\text { C. K. Schneid. }\end{array}$ & $0 \pm 0$ & $24.7 \pm 3.26$ & $0 \pm 0$ & $18.7 \pm 1.76$ & $9.8 \pm 0.65$ & $16.3 \pm 1.52$ & $12.4 \pm 1.21$ & $0 \pm 0$ \\
\hline Ranunculaceae & Clematis flammula $\mathrm{L}$. & $10.6 \pm 1.44$ & $24.6 \pm 2.87$ & $1.3 \pm 0.13$ & $15.4 \pm 1.72$ & $2.7 \pm 0.32$ & $17.8 \pm 1.72$ & $9.3 \pm 0.94$ & $0 \pm 0$ \\
\hline Rosaceae & Prunus dulcis (Mill.) D. A. Webb & $0 \pm 0$ & $26.1 \pm 3.54$ & $0 \pm 0$ & $16.6 \pm 1.76$ & $10.5 \pm 1.23$ & $16.3 \pm 1.61$ & $1.2 \pm 0.11$ & $0 \pm 0$ \\
\hline Rosaceae & Prunus laurocerasus L. & $10.2 \pm 1.23$ & $23.8 \pm 1.97$ & $2.4 \pm 0.32$ & $18.1 \pm 1.89$ & $12.7 \pm 1.45$ & $16.4 \pm 1.62$ & $1.3 \pm 0.18$ & $0 \pm 0$ \\
\hline Rutaceae & Dictamnus alba $\mathrm{L}$. & $9.8 \pm 1.24$ & $25.8 \pm 2.79$ & $2.8 \pm 0.23$ & $17.8 \pm 1.89$ & $5.8 \pm 0.43$ & $15.3 \pm 1.41$ & $6.4 \pm 0.34$ & $0 \pm 0$ \\
\hline Rutaceae & Phellodendron amurense Rupr. & $4.3 \pm 0.56$ & $23.8 \pm 2.86$ & $0 \pm 0$ & $16.6 \pm 1.78$ & $2.3 \pm 0.23$ & $17.6 \pm 1.56$ & $3.3 \pm 0.32$ & $0 \pm 0$ \\
\hline Rutaceae & Ptelea trifoliata $\mathrm{L}$. & $4.3 \pm 0.89$ & $27.7 \pm 4.24$ & $0 \pm 0$ & $18.2 \pm 1.57$ & $0 \pm 0$ & $17.6 \pm 1.49$ & $0 \pm 0$ & $0 \pm 0$ \\
\hline Sapindaceae & Koelreuteria paniculata Laxm. & $8.4 \pm 1.21$ & $26.7 \pm 4.12$ & $0 \pm 0$ & $17.8 \pm 1.15$ & $11.5 \pm 1.45$ & $13.9 \pm 1.74$ & $8.9 \pm 0.84$ & $0 \pm 0$ \\
\hline Simaroubaceae & Ailanthus altissima (Mill.) Swingle & $2.2 \pm 0.56$ & $24.7 \pm 2.87$ & $1.3 \pm 0.16$ & $16.7 \pm 1.69$ & $3.4 \pm 0.32$ & $15.8 \pm 1.54$ & $10.4 \pm 0.92$ & $0 \pm 0$ \\
\hline Tamaricaceae & Tamarix elongata Ledeb. & $2.1 \pm 0.35$ & $26.4 \pm 3.31$ & $1.6 \pm 0.16$ & $15.9 \pm 1.69$ & $0 \pm 0$ & $15.4 \pm 1.52$ & $7.7 \pm 0.56$ & $0 \pm 0$ \\
\hline Vitaceae & $\begin{array}{l}\text { Parthenocissus tricuspidata } \\
\text { (Siebold \& Zucc.) Planch. }\end{array}$ & $0 \pm 0$ & $24.9 \pm 2.98$ & $3.6 \pm 0.17$ & $17.8 \pm 1.88$ & $4.7 \pm 0.32$ & $16.4 \pm 1.52$ & $13.6 \pm 1.45$ & $0 \pm 0$ \\
\hline
\end{tabular}

Note: *-disks with $15.0 \mu \mathrm{g}$ of azithromycin were used for all bacteria as positive control. 
Table 4

Antibacterial effect of ethanol extracts of plants on Serratia marcescens, Yersinia enterocolitica, Klebsiella pneumoniae and Salmonella typhimurium ( $\mathrm{x} \pm \mathrm{SD}, \mathrm{n}=8$ )

\begin{tabular}{|c|c|c|c|c|c|c|c|c|c|}
\hline \multirow{2}{*}{ Family } & \multirow{2}{*}{ Species } & \multicolumn{2}{|c|}{ S. marcescens } & \multicolumn{2}{|c|}{ Y. enterocolitica } & \multicolumn{2}{|c|}{ K. pneumoniae } & \multicolumn{2}{|c|}{ S.typhimurium } \\
\hline & & test & control $^{*}$ & test & control $^{*}$ & test & control $^{*}$ & test & control $^{*}$ \\
\hline Aristolochiaceae & Aristolochia manshuriensis Kom. & $0 \pm 0$ & $0 \pm 0$ & $0 \pm 0$ & $14.4 \pm 1.42$ & $0 \pm 0$ & $0 \pm 0$ & $2.6 \pm 0.32$ & $19.7 \pm 1.45$ \\
\hline Asparagaceae & Polygonatum multiflorum (L.) All. & $7.8 \pm 0.77$ & $0 \pm 0$ & $11.4 \pm 1.21$ & $14.9 \pm 1.44$ & $0 \pm 0$ & $0 \pm 0$ & $4.4 \pm 0.87$ & $21.7 \pm 1.67$ \\
\hline Asteraceae & Artemisia absinthium L. & $2.8 \pm 0.21$ & $0 \pm 0$ & $0 \pm 0$ & $14.7 \pm 1.69$ & $4.1 \pm 0.32$ & $0 \pm 0$ & $4.3 \pm 0.44$ & $22.6 \pm 1.75$ \\
\hline Berberidaceae & Berberis vulgaris L. & $12.6 \pm 1.34$ & $0 \pm 0$ & $0 \pm 0$ & $14.8 \pm 1.51$ & $9.3 \pm 0.89$ & $0 \pm 0$ & $10.6 \pm 1.21$ & $21.7 \pm 1.54$ \\
\hline Bignoniaceae & Campsis radicans (L.) Seem. & $0 \pm 0$ & $0 \pm 0$ & $2.7 \pm 0.21$ & $15.8 \pm 1.73$ & $1.6 \pm 0.16$ & $0 \pm 0$ & $12.6 \pm 1.12$ & $21.5 \pm 2.31$ \\
\hline Bignoniaceae & Catalpa duclouxii Dode & $2.4 \pm 0.21$ & $0 \pm 0$ & $0 \pm 0$ & $13.3 \pm 1.81$ & $10.7 \pm 0.89$ & $0 \pm 0$ & $0 \pm 0$ & $22.3 \pm 2.19$ \\
\hline Calycanthaceae & Chimonanthus praecox (L.) Link & $8.2 \pm 0.67$ & $0 \pm 0$ & $2.9 \pm 0.32$ & $13.4 \pm 1.62$ & $4.3 \pm 0.32$ & $0 \pm 0$ & $0 \pm 0$ & $22.7 \pm 2.64$ \\
\hline Celastraceae & Celastrus scandens $\mathrm{L}$. & $3.7 \pm 0.24$ & $0 \pm 0$ & $0 \pm 0$ & $13.1 \pm 1.48$ & $0 \pm 0$ & $0 \pm 0$ & $4.4 \pm 0.21$ & $19.6 \pm 2.11$ \\
\hline Colchicaceae & Colchicum autumnale L. & $10.8 \pm 1.43$ & $0 \pm 0$ & $6.6 \pm 0.54$ & $14.7 \pm 1.39$ & $12.8 \pm 1.45$ & $0 \pm 0$ & $2.4 \pm 0.22$ & $22.7 \pm 2.57$ \\
\hline Dennstaedtiaceae & Pteridium aquilinum (L.) Kuhn & $1.8 \pm 0.21$ & $0 \pm 0$ & $8.2 \pm 0.78$ & $15.4 \pm 1.38$ & $2.3 \pm 0.32$ & $0 \pm 0$ & $2.4 \pm 0.32$ & $19.8 \pm 1.45$ \\
\hline Eucommiaceae & Eucommia ulmoides Oliv. & $0 \pm 0$ & $0 \pm 0$ & $0 \pm 0$ & $14.7 \pm 1.71$ & $0 \pm 0$ & $0 \pm 0$ & $0 \pm 0$ & $19.7 \pm 2.19$ \\
\hline Fabaceae & Genista tinctoria $\mathrm{L}$. & $7.6 \pm 0.76$ & $0 \pm 0$ & $0 \pm 0$ & $15.2 \pm 1.67$ & $9.3 \pm 0.87$ & $0 \pm 0$ & $9.4 \pm 1.12$ & $23.6 \pm 2.44$ \\
\hline Fabaceae & Laburnum anagyroides Medik. & $1.6 \pm 0.13$ & $0 \pm 0$ & $0 \pm 0$ & $12.4 \pm 1.77$ & $3.2 \pm 0.34$ & $0 \pm 0$ & $1.9 \pm 0.33$ & $20.5 \pm 1.87$ \\
\hline Fabaceae & Securigera varia $(\mathrm{L}$.$) Lassen$ & $0 \pm 0$ & $0 \pm 0$ & $0 \pm 0$ & $14.9 \pm 1.48$ & $1.5 \pm 0.21$ & $0 \pm 0$ & $0 \pm 0$ & $21.3 \pm 1.76$ \\
\hline Fabaceae & Styphnolobium japonicum (L.) Schott & $2.3 \pm 0.19$ & $0 \pm 0$ & $0 \pm 0$ & $15.9 \pm 0.98$ & $0 \pm 0$ & $0 \pm 0$ & $0 \pm 0$ & $19.6 \pm 1.65$ \\
\hline Fabaceae & Wisteria sinensis (Sims) Sweet & $3.4 \pm 0.23$ & $0 \pm 0$ & $0 \pm 0$ & $13.4 \pm 1.39$ & $0 \pm 0$ & $0 \pm 0$ & $8.7 \pm 0.98$ & $21.8 \pm 1.98$ \\
\hline Fagaceae & Quercus castaneifolia C. A. Mey. & $0 \pm 0$ & $0 \pm 0$ & $11.8 \pm 1.35$ & $15.3 \pm 1.48$ & $10.5 \pm 1.23$ & $0 \pm 0$ & $0 \pm 0$ & $21.8 \pm 1.98$ \\
\hline Fagaceae & $\begin{array}{l}\text { Q. petraea iberica } \\
\text { (Steven ex M. Bieb.) Krassiln. }\end{array}$ & $0 \pm 0$ & $0 \pm 0$ & $0 \pm 0$ & $14.6 \pm 1.49$ & $10.4 \pm 1.21$ & $0 \pm 0$ & $0 \pm 0$ & $22.5 \pm 2.31$ \\
\hline Geraniaceae & Geranium sanguineum L. & $2.3 \pm 0.42$ & $0 \pm 0$ & $2.7 \pm 0.19$ & $12.7 \pm 1.83$ & $0 \pm 0$ & $0 \pm 0$ & $1.7 \pm 0.34$ & $19.2 \pm 1.87$ \\
\hline Ginkgoaceae & Ginkgo biloba L. & $11.6 \pm 1.34$ & $0 \pm 0$ & $9.2 \pm 0.76$ & $12.3 \pm 1.74$ & $25.5 \pm 2.78$ & $0 \pm 0$ & $2.2 \pm 0.17$ & $23.8 \pm 2.45$ \\
\hline Lamiaceae & Callicarpa bodinieri H. Lév. & $2.8 \pm 0.18$ & $0 \pm 0$ & $0 \pm 0$ & $13.5 \pm 1.22$ & $0 \pm 0$ & $0 \pm 0$ & $1.5 \pm 0.23$ & $20.7 \pm 2.12$ \\
\hline Lamiaceae & Nepeta racemosa Lam. & $1.7 \pm 0.12$ & $0 \pm 0$ & $0 \pm 0$ & $14.5 \pm 1.73$ & $1.8 \pm 0.17$ & $0 \pm 0$ & $2.4 \pm 0.31$ & $23.8 \pm 2.76$ \\
\hline Lamiaceae & Salvia officinalis L. & $2.4 \pm 0.26$ & $0 \pm 0$ & $0 \pm 0$ & $12.7 \pm 1.64$ & $10.7 \pm 0.78$ & $0 \pm 0$ & $0 \pm 0$ & $21.7 \pm 2.34$ \\
\hline Lamiaceae & Vitex agnus-castus $\mathrm{L}$. & $10.8 \pm 0.89$ & $0 \pm 0$ & $1.2 \pm 0.12$ & $12.4 \pm 1.64$ & $1.6 \pm 0.11$ & $0 \pm 0$ & $9.7 \pm 0.89$ & $23.4 \pm 2.45$ \\
\hline Lamiaceae & Vitex negundo L. & $3.2 \pm 0.36$ & $0 \pm 0$ & $2.2 \pm 0.16$ & $13.7 \pm 1.37$ & $16.7 \pm 1.21$ & $0 \pm 0$ & $10.7 \pm 0.96$ & $21.6 \pm 1.89$ \\
\hline Magnoliaceae & Liriodendron tulipifera $\mathrm{L}$. & $2.4 \pm 0.19$ & $0 \pm 0$ & $1.2 \pm 0.14$ & $15.5 \pm 1.78$ & $25.4 \pm 2.77$ & $0 \pm 0$ & $2.1 \pm 0.21$ & $20.7 \pm 1.99$ \\
\hline Magnoliaceae & Magnolia kobus DC. & $9.8 \pm 0.93$ & $0 \pm 0$ & $0 \pm 0$ & $15.2 \pm 1.39$ & $0 \pm 0$ & $0 \pm 0$ & $8.7 \pm 1.67$ & $22.3 \pm 2.19$ \\
\hline Moraceae & $\begin{array}{l}\text { Machura pomifera (Raf.) } \\
\text { C. K. Schneid. }\end{array}$ & $19.4 \pm 1.78$ & $0 \pm 0$ & $10.7 \pm 0.87$ & $13.3 \pm 1.72$ & $3.2 \pm 0.41$ & $0 \pm 0$ & $10.5 \pm 1.21$ & $18.7 \pm 2.14$ \\
\hline Ranunculaceae & Clematis flammula $\mathrm{L}$. & $5.4 \pm 0.34$ & $0 \pm 0$ & $0 \pm 0$ & $14.8 \pm 1.72$ & $0 \pm 0$ & $0 \pm 0$ & $1.2 \pm 0.43$ & $20.6 \pm 1.89$ \\
\hline Rosaceae & Prunus dulcis (Mill.) D. A.Webb & $3.4 \pm 0.45$ & $0 \pm 0$ & $7.2 \pm 0.78$ & $15.3 \pm 1.81$ & $0 \pm 0$ & $0 \pm 0$ & $5.2 \pm 0.87$ & $19.1 \pm 1.98$ \\
\hline Rosaceae & Prunus laurocerasus L. & $0 \pm 0$ & $0 \pm 0$ & $1.1 \pm 0.11$ & $14.4 \pm 1.52$ & $0 \pm 0$ & $0 \pm 0$ & $2.6 \pm 0.21$ & $21.8 \pm 2.31$ \\
\hline Rutaceae & Dictamnus alba $\mathrm{L}$. & $0 \pm 0$ & $0 \pm 0$ & $7.8 \pm 0.88$ & $14.3 \pm 1.71$ & $0 \pm 0$ & $0 \pm 0$ & $0 \pm 0$ & $18.8 \pm 1.85$ \\
\hline Rutaceae & Phellodendron amurense Rupr. & $14.2 \pm 1.18$ & $0 \pm 0$ & $2.2 \pm 0.23$ & $13.6 \pm 1.96$ & $6.3 \pm 0.56$ & $0 \pm 0$ & $10.8 \pm 1.18$ & $19.6 \pm 2.17$ \\
\hline Rutaceae & Ptelea trifoliata $\mathrm{L}$. & $0 \pm 0$ & $0 \pm 0$ & $2.7 \pm 0.28$ & $15.6 \pm 1.79$ & $0 \pm 0$ & $0 \pm 0$ & $2.2 \pm 0.14$ & $22.7 \pm 2.54$ \\
\hline Sapindaceae & Koelreuteria paniculata Laxm. & $16.7 \pm 1.22$ & $0 \pm 0$ & $4.5 \pm 0.31$ & $12.9 \pm 1.34$ & $0 \pm 0$ & $0 \pm 0$ & $10.7 \pm 0.97$ & $18.7 \pm 1.86$ \\
\hline Simaroubaceae & Ailanthus altissima (Mill.) Swingle & $2.1 \pm 0.18$ & $0 \pm 0$ & $0 \pm 0$ & $14.8 \pm 1.64$ & $0 \pm 0$ & $0 \pm 0$ & $1.4 \pm 0.43$ & $19.7 \pm 1.87$ \\
\hline Tamaricaceae & Tamarix elongata Ledeb. & $3.5 \pm 0.34$ & $0 \pm 0$ & $3.3 \pm 0.41$ & $15.4 \pm 1.52$ & $0 \pm 0$ & $0 \pm 0$ & $0 \pm 0$ & $22.4 \pm 2.11$ \\
\hline Vitaceae & $\begin{array}{l}\text { Parthenocissus tricuspidata } \\
\text { (Siebold \& Zucc.) Planch. }\end{array}$ & $13.8 \pm 1.31$ & $0 \pm 0$ & $4.6 \pm 0.24$ & $15.4 \pm 1.52$ & $0 \pm 0$ & $0 \pm 0$ & $3.4 \pm 0.19$ & $22.9 \pm 2.31$ \\
\hline
\end{tabular}

Note: see Table 3 .

Two poly-resistant strains of microorganisms of Yersiniaceae and Enterobacteriaceae families - Serratia marcescens and Klebsiella pneumoniae - were determined to have completely no sensitivity to azithromycin. At the same time, we determined ethanol extracts of Maclura pomifera (19.4), Koelreuteria paniculata (16.7), Phellodendron amurense (14.2), Parthenocissus tricuspidata (13.8), Berberis vulgaris (12.6), Ginkgo biloba (11.6), Vitex agnus-castus (10.8) and Colchicum autumnale (10.8) to be highly effective in arresting the growth of $S$. marcescens bacteria. Three extracts - Quercus castaneifolia, Polygonatum multiflorum and Maclura pomifera - had high inhibiting effect on $Y$. enterocolitica $(11.8,11.4,10.7)$, whereas Ginkgo biloba (25.5), Liriodendron tulipifera (25.4), Vitex negundo (16.7), Colchicum autumnale (12.8), Salvia officinalis (10.7), Catalpa duclouxii (10.7), Quercus castaneifolia (10.5) and Quercus petraea (10.4) were effective against $K$. pneumoniae. Extracts from Ginkgo biloba and Liriodendron tulipifera produced 1.5-2.4-fold greater growth inhibition zones of colonies of $K$. pneumoniae than other species of plants.

We also saw high inhibiting impact on the colonies of S. typhimurium bacteria exerted by alcohol extracts of Campsis radicans (12.6), Phellodendron amurense (10.8), Vitex negundo (10.7), Koelreuteria paniculata (10.7), Berberis vulgaris (10.6) and Maclura pomifera (10.5) at moderate zones of growth inhibition produced by Vitex agnus-castus (9.7), Genista tinctoria (9.4), Wisteria sinensis (8.7) and Magnolia kobus (8.7).

Interesting results were obtained for use of ethanol extracts against microorganisms of the Listeriaceae family (Table 5). While $L$. monocytogenes was highly susceptible to 8 plants (Ptelea trifoliata
(15.8), Clematis flammula (13.5), Aristolochia manshuriensis (10.8), Tamarix elongata (10.8), Colchicum autumnale (10.7), Wisteria sinensis (10.4), Ginkgo biloba (10.4), Chimonanthus praecox (10.1)), L. innocua - to 5 (Ginkgo biloba (19.7), Liriodendron tulipifera (12.3), Geranium sanguineum (11.2), Securigera varia (10.7), Prunus laurocerasus (10.4)), and L. ivanovi was susceptible only to ethanol extract of Colchicum autumnale (10.8).

During our research we determined that some plants arrest the growth of colonies of $P$. aeruginosa: Ginkgo biloba (21.3), Berberis vulgaris (16.8), Liriodendron tulipifera (14.3), Geranium sanguineum (10.8), Genista tinctoria (10.7), Clematis flammula (10.4), Colchicum autumnale (10.4) and Tamarix elongata (10.3). Ethanol extract of Ginkgo biloba exceeded the other tested plants regarding the width of growth inhibition zone of $P$. aeruginosa by $1.3-2.1$ times. We determined tolerance of the tested strains of $L$. monocytogenes and $P$. aeruginosa strains to the action of azithromycin.

High antibacterial activities (Table 6) against $C$. jejuni was confirmed for ten ethanol extracts (Ptelea trifoliata (17.5), Quercus petraea iberica (16.2), Koelreuteria paniculata (13.3), Tamarix elongata (11.4), Geranium sanguineum (11.3), Magnolia kobus (10.8), Callicarpa bodinieri (10.7), Maclura pomifera (10.5), Clematis flammula (10.3) and Liriodendron tulipifera (10.3)), and moderate activity for five plants (Phellodendron amurense (9.7), Berberis vulgaris (9.4), Chimonanthus praecox (8.7), Ginkgo biloba (8.4) and Salvia officinalis (8.2)). We observed the strain of $C$. jejuni to be resistant to azithromycin. Also, high 
inhibiting power against $R h$. equi was displayed by fifteen tested alcohol extracts: Campsis radicans (18.6), Prumus laurocerasus (16.5), Vitex negundo (13.4), Phellodendron amurense (12.9), Genista tinctoria (12.8), Liriodendron tulipifera (11.8), Tamarix elongata (11.4), Pteridium aquilinum (11.4), Salvia officinalis (11.2), Maclura pomifera (10.8),
Callicarpa bodinieri (10.8), Prunus dulcis (10.6), Geranium sanguineum (10.4), Ptelea trifoliata (10.3) and Catalpa duclouxii (10.2). We found five alcohol extracts with maximum antibacterial impacts on $C$. xerosis: Maclura pomifera (10.9), Vitex agmus-castus (10.6), Clematis flammula (10.5), Artemisia absinthium (10.2) and Phellodendron amurense (10.2).

Table 5

Antibacterial effect of ethanol extracts of plants on bacteria of families Listeriaceae (Listeria ivanovii, L. innocua, L. monocytogenes) and Pseudomonadaceae (Pseudomonas aeruginosa) $(\mathrm{x} \pm \mathrm{SD}, \mathrm{n}=8)$

\begin{tabular}{|c|c|c|c|c|c|c|c|c|c|}
\hline \multirow{2}{*}{ Family } & \multirow{2}{*}{ Species } & \multicolumn{2}{|c|}{ L. ivanovi } & \multicolumn{2}{|c|}{ L. iпnосиа } & \multicolumn{2}{|c|}{ L. monocytogenes } & \multicolumn{2}{|c|}{ P. aeruginosa } \\
\hline & & test & control $^{*}$ & test & control $^{*}$ & test & control $^{*}$ & test & control \\
\hline Aristolochiaceae & Aristolochia manshuriensis Kom. & $0 \pm 0$ & $14.3 \pm 1.54$ & $0 \pm 0$ & $28.7 \pm 3.44$ & $10.8 \pm 1.33$ & $0 \pm 0$ & $8.7 \pm 0.98$ & $0 \pm 0$ \\
\hline Asparagaceae & Polygonatum multiflorum (L.) All. & $0 \pm 0$ & $15.9 \pm 1.67$ & $0 \pm 0$ & $25.7 \pm 2.31$ & $0 \pm 0$ & $0 \pm 0$ & $5.4 \pm 0.76$ & $0 \pm 0$ \\
\hline Asteraceae & Artemisia absinthium L. & $0 \pm 0$ & $15.9 \pm 1.61$ & $0 \pm 0$ & $26.6 \pm 2.31$ & $0 \pm 0$ & $0 \pm 0$ & $1.7 \pm 0.21$ & $0 \pm 0$ \\
\hline Berberidaceae & Berberis vulgaris L. & $0 \pm 0$ & $17.7 \pm 1.65$ & $4.3 \pm 0.45$ & $26.7 \pm 1.87$ & $0 \pm 0$ & $0 \pm 0$ & $16.8 \pm 1.31$ & $0 \pm 0$ \\
\hline Bignoniaceae & Campsis radicans (L.) Seem. & $0 \pm 0$ & $16.6 \pm 1.85$ & $0 \pm 0$ & $27.5 \pm 3.22$ & $0 \pm 0$ & $0 \pm 0$ & $2.1 \pm 0.32$ & $0 \pm 0$ \\
\hline Bignoniaceae & Catalpa duclouxii Dode & $0 \pm 0$ & $17.5 \pm 1.87$ & $0 \pm 0$ & $26.3 \pm 1.87$ & $8.2 \pm 0.88$ & $0 \pm 0$ & $0 \pm 0$ & $0 \pm 0$ \\
\hline Calycanthaceae & Chimonanthus praecox (L.) Link & $0 \pm 0$ & $17.4 \pm 1.66$ & $0 \pm 0$ & $27.7 \pm 3.11$ & $10.1 \pm 0.88$ & $0 \pm 0$ & $0 \pm 0$ & $0 \pm 0$ \\
\hline Celastraceae & Celastrus scandens L. & $0 \pm 0$ & $15.4 \pm 0.79$ & $0 \pm 0$ & $26.6 \pm 2.78$ & $0 \pm 0$ & $0 \pm 0$ & $6.3 \pm 0.66$ & $0 \pm 0$ \\
\hline Colchicaceae & Colchicum autumnale L. & $10.8 \pm 0.86$ & $16.8 \pm 1.28$ & $0 \pm 0$ & $27.7 \pm 2.68$ & $10.7 \pm 0.99$ & $0 \pm 0$ & $10.4 \pm 1.14$ & $0 \pm 0$ \\
\hline Dennstaedtiaceae & Pteridium aquilinum (L.) Kuhn & $2.7 \pm 0.19$ & $17.4 \pm 1.31$ & $0 \pm 0$ & $26.8 \pm 2.78$ & $0 \pm 0$ & $0 \pm 0$ & $6.8 \pm 0.77$ & $0 \pm 0$ \\
\hline Eucommiaceae & Eucommia ulmoides Oliv. & $0 \pm 0$ & $15.8 \pm 1.11$ & $0 \pm 0$ & $29.7 \pm 3.21$ & $0 \pm 0$ & $0 \pm 0$ & $1.5 \pm 0.16$ & $0 \pm 0$ \\
\hline Fabaceae & Genista tinctoria $\mathrm{L}$. & $0 \pm 0$ & $18.8 \pm 1.31$ & $0 \pm 0$ & $28.6 \pm 3.12$ & $0 \pm 0$ & $0 \pm 0$ & $10.7 \pm 1.21$ & $0 \pm 0$ \\
\hline Fabaceae & Laburnum anagyroides Medik. & $0 \pm 0$ & $14.2 \pm 1.62$ & $0 \pm 0$ & $28.5 \pm 2.88$ & $0 \pm 0$ & $0 \pm 0$ & $0 \pm 0$ & $0 \pm 0$ \\
\hline Fabaceae & Securigera varia (L.) Lassen & $0 \pm 0$ & $17.8 \pm 1.19$ & $10.7 \pm 1.08$ & $27.3 \pm 1.89$ & $0 \pm 0$ & $0 \pm 0$ & $0 \pm 0$ & $0 \pm 0$ \\
\hline Fabaceae & $\begin{array}{l}\text { Styphnolobium japonicum } \\
\text { (L.) Schott }\end{array}$ & $0 \pm 0$ & $16.6 \pm 0.87$ & $0 \pm 0$ & $27.6 \pm 2.78$ & $0 \pm 0$ & $0 \pm 0$ & $2.3 \pm 0.32$ & $0 \pm 0$ \\
\hline Fabaceae & Wisteria sinensis (Sims) Sweet & $0 \pm 0$ & $16.7 \pm 1.74$ & $0 \pm 0$ & $27.8 \pm 2.76$ & $10.4 \pm 1.33$ & $0 \pm 0$ & $4.2 \pm 0.31$ & $0 \pm 0$ \\
\hline Fagaceae & Quercus castaneifolia C. A. Mey. & $0 \pm 0$ & $15.5 \pm 1.89$ & $0 \pm 0$ & $29.8 \pm 4.01$ & $0 \pm 0$ & $0 \pm 0$ & $0 \pm 0$ & $0 \pm 0$ \\
\hline Fagaceae & $\begin{array}{l}\text { Q. petraea iberica } \\
\text { (Steven ex M. Bieb.) Krassiln. }\end{array}$ & $0 \pm 0$ & $17.5 \pm 1.32$ & $0 \pm 0$ & $26.5 \pm 2.78$ & $4.2 \pm 0.67$ & $0 \pm 0$ & $0 \pm 0$ & $0 \pm 0$ \\
\hline Geraniaceae & Geranium sanguineum L. & $0 \pm 0$ & $17.7 \pm 1.56$ & $11.2 \pm 0.78$ & $28.2 \pm 2.89$ & $5.3 \pm 0.44$ & $0 \pm 0$ & $10.8 \pm 1.21$ & $0 \pm 0$ \\
\hline Ginkgoaceae & Ginkgo biloba L. & $0 \pm 0$ & $16.3 \pm 1.44$ & $19.7 \pm 1.31$ & $27.8 \pm 3.21$ & $10.4 \pm 1.26$ & $0 \pm 0$ & $21.3 \pm 2.13$ & $0 \pm 0$ \\
\hline Lamiaceae & Callicarpa bodinieri H. Lév. & $0 \pm 0$ & $15.5 \pm 1.21$ & $0 \pm 0$ & $27.7 \pm 2.56$ & $0 \pm 0$ & $0 \pm 0$ & $8.5 \pm 0.87$ & $0 \pm 0$ \\
\hline Lamiaceae & Nepeta racemosa Lam. & $0 \pm 0$ & $16.5 \pm 1.32$ & $0 \pm 0$ & $26.8 \pm 2.77$ & $0 \pm 0$ & $0 \pm 0$ & $0 \pm 0$ & $0 \pm 0$ \\
\hline Lamiaceae & Salvia officinalis L. & $0 \pm 0$ & $16.8 \pm 1.11$ & $0 \pm 0$ & $26.7 \pm 2.18$ & $0 \pm 0$ & $0 \pm 0$ & $8.4 \pm 0.78$ & $0 \pm 0$ \\
\hline Lamiaceae & Vitex agnus-castus L. & $0 \pm 0$ & $16.3 \pm 0.96$ & $0 \pm 0$ & $26.4 \pm 2.56$ & $0 \pm 0$ & $0 \pm 0$ & $4.2 \pm 0.65$ & $0 \pm 0$ \\
\hline Lamiaceae & Vitex negundo L. & $0 \pm 0$ & $18.9 \pm 1.75$ & $0 \pm 0$ & $27.3 \pm 2.51$ & $0 \pm 0$ & $0 \pm 0$ & $0 \pm 0$ & $0 \pm 0$ \\
\hline Magnoliaceae & Liriodendron tulipifera $\mathrm{L}$. & $0 \pm 0$ & $17.7 \pm 1.34$ & $12.3 \pm 2.11$ & $29.7 \pm 4.02$ & $0 \pm 0$ & $0 \pm 0$ & $14.3 \pm 0.88$ & $0 \pm 0$ \\
\hline Magnoliaceae & Magnolia kobus DC. & $0 \pm 0$ & $16.5 \pm 1.33$ & $0 \pm 0$ & $25.3 \pm 2.20$ & $0 \pm 0$ & $0 \pm 0$ & $0 \pm 0$ & $0 \pm 0$ \\
\hline Moraceae & $\begin{array}{l}\text { Machura pomifera (Raf.) } \\
\text { C. K. Schneid. }\end{array}$ & $0 \pm 0$ & $14.7 \pm 1.32$ & $0 \pm 0$ & $27.7 \pm 3.29$ & $0 \pm 0$ & $0 \pm 0$ & $2.2 \pm 0.43$ & $0 \pm 0$ \\
\hline Ranunculaceae & Clematis flammula $\mathrm{L}$ & $0 \pm 0$ & $14.4 \pm 1.77$ & $0 \pm 0$ & $27.6 \pm 2.31$ & $13.5 \pm 1.77$ & $0 \pm 0$ & $10.4 \pm 1.33$ & $0 \pm 0$ \\
\hline Rosaceae & Prunus dulcis (Mill.) D. A. Webb & $0 \pm 0$ & $17.6 \pm 1.43$ & $0 \pm 0$ & $27.1 \pm 2.78$ & $0 \pm 0$ & $0 \pm 0$ & $2.5 \pm 0.21$ & $0 \pm 0$ \\
\hline Rosaceae & Prunus laurocerasus L. & $0 \pm 0$ & $17.1 \pm 1.42$ & $10.4 \pm 1.67$ & $27.8 \pm 2.33$ & $0 \pm 0$ & $0 \pm 0$ & $2.8 \pm 0.33$ & $0 \pm 0$ \\
\hline Rutaceae & Dictammus alba L. & $0 \pm 0$ & $17.8 \pm 1.22$ & $0 \pm 0$ & $26.8 \pm 2.44$ & $0 \pm 0$ & $0 \pm 0$ & $1.1 \pm 0.18$ & $0 \pm 0$ \\
\hline Rutaceae & Phellodendron amurense Rupr. & $0 \pm 0$ & $15.6 \pm 1.31$ & $0 \pm 0$ & $29.6 \pm 3.65$ & $0 \pm 0$ & $0 \pm 0$ & $0 \pm 0$ & $0 \pm 0$ \\
\hline Rutaceae & Ptelea trifoliata L. & $0 \pm 0$ & $16.2 \pm 1.33$ & $0 \pm 0$ & $28.7 \pm 3.42$ & $15.4 \pm 1.37$ & $0 \pm 0$ & $2.4 \pm 0.21$ & $0 \pm 0$ \\
\hline Sapindaceae & Koelreuteria paniculata Laxm. & $0 \pm 0$ & $17.8 \pm 0.97$ & $0 \pm 0$ & $28.7 \pm 3.27$ & $0 \pm 0$ & $0 \pm 0$ & $0 \pm 0$ & $0 \pm 0$ \\
\hline Simaroubaceae & Ailanthus altissima (Mill.) Swingle & $0 \pm 0$ & $15.7 \pm 1.87$ & $0 \pm 0$ & $25.7 \pm 1.97$ & $9.1 \pm 1.27$ & $0 \pm 0$ & $0 \pm 0$ & $0 \pm 0$ \\
\hline Tamaricaceae & Tamarix elongata Ledeb. & $0 \pm 0$ & $17.9 \pm 1.65$ & $0 \pm 0$ & $27.4 \pm 2.34$ & $10.8 \pm 1.23$ & $0 \pm 0$ & $10.3 \pm 0.98$ & $0 \pm 0$ \\
\hline Vitaceae & $\begin{array}{l}\text { Parthenocissus tricuspidata } \\
\text { (Siebold \& Zucc.) Planch. }\end{array}$ & $4.3 \pm 0.19$ & $15.8 \pm 1.76$ & $0 \pm 0$ & $26.9 \pm 2.13$ & $0 \pm 0$ & $0 \pm 0$ & $0 \pm 0$ & $0 \pm 0$ \\
\hline
\end{tabular}

Note: see Table 3.

Antifungal impact (Table 6) on C. albicans with inhibition zone of over $8 \mathrm{~mm}$ was exerted by five extracts: Artemisia absinthium (11.7), Parthenocissus tricuspidata (10.7), Liriodendron tulipifera (9.4), Phellodendron amurense (8.7) and Celastrus scandens (8.4), which was several-fold higher than the control (amphotericin), for which the growth inhibition zone of $C$. albicans ranged $2.1-2.4 \mathrm{~mm}$.

\section{Discussion}

Substances produced by Embryophyta as secondary metabolites were found to be biologically quite active compounds against microorganisms pathogenic for humans and agricultural animals. Some of the plants we studied may become the basis for the development of new pharmaceutical preparations (Zazharskyi et al., 2019c).

Kavitha \& Nelson (2016) consider that chloroform extract of leaves of Aristolochia manshuriensis will become the alternative for the treatment of threat of pathogenic organisms. The authors found twenty bioactive constituents and functional groups associated with ethanol, carbonic acid, alkanes, aldehides, aroma acids present in chloroform extract of leaves of A. manshuriensis. The studied extract inhibited Vibrio harveyi, $V$. vulnificus and Serratia marcescens. Hydroethanol extract from Celastrus scandens L., against the background of high antioxidant activity (2,2-diphenyl-1-picrylhydrazyl, chelation of metals, capabilities to restore three-valent iron in plasm, superoxide radical and nitrogen oxide), good anti-inflammatory activity, displayed low antibacterial and antifungal properties (Kumar \& Sharma, 2018).

Artemisia absinthium L. are perennial plants with ubiquitous distribution in deserts and dry places of Eurasia, usually growing on slopes of hills, sides of the roads and fields. It is native to Europe, North Asia and North Africa. The plant can contain toxic substances (thujon for example) responsible for side effects. Absinthe is used in phytotherapy due to its tonic, spasmolytic, antipyretic and anthelmintic properties. Obistioiu \& Chiurciu (2014) and Al-Ghamdi (2020) report fungicidal effect of A. absinthium.

The structure of Berberis vulgaris L. contains some polyphenolic, alkaloid compounds which hinder the activity of bacteria (Özgen \& Geçer, 2012). Nanocrystals of zinc oxide prepared using $B$. vulgaris exhibited significant antibacterial activity against S. aureus (Anzabi, 2018). 
Table 6

Antibacterial effect of extracts of plants on bacteria Campylobacter jejuni, Rhodococcus equi, Corynebacterium xerosis and fungus Candida albicans $(\mathrm{x} \pm \mathrm{SD}, \mathrm{n}=8$ )

\begin{tabular}{|c|c|c|c|c|c|c|c|c|c|}
\hline \multirow{2}{*}{ Family } & \multirow{2}{*}{ Species } & \multicolumn{2}{|c|}{ C. jejuni } & \multicolumn{2}{|c|}{ Rh. equi } & \multicolumn{2}{|c|}{ C.xerosis } & \multicolumn{2}{|c|}{ C. albicans } \\
\hline & & test & control $^{*}$ & test & control $^{*}$ & test & control $^{*}$ & test & control $^{* *}$ \\
\hline Aristolochiaceae & Aristolochia manshuriensis Kom. & $2.5 \pm 0.21$ & $0 \pm 0$ & $3.1 \pm 0.31$ & $20.7 \pm 3.44$ & $9.3 \pm 0.96$ & $10.7 \pm 2.67$ & $3.7 \pm 0.33$ & $0 \pm 0 ; 2.4 \pm 0.20$ \\
\hline Asparagaceae & Polygonatum multiflorum (L.) All. & $0 \pm 0$ & $0 \pm 0$ & $0 \pm 0$ & $23.7 \pm 2.31$ & $2.2 \pm 0.12$ & $12.7 \pm 1.56$ & $2.4 \pm 0.22$ & $0 \pm 0 ; 2.2 \pm 0.15$ \\
\hline Asteraceae & Artemisia absinthium L. & $0 \pm 0$ & $0 \pm 0$ & $9.3 \pm 0.91$ & $23.6 \pm 2.31$ & $10.2 \pm 0.89$ & $11.6 \pm 1.98$ & $11.7 \pm 1.23$ & $0 \pm 0 ; 2.1 \pm 0.15$ \\
\hline Berberidaceae & Berberis vulgaris $\mathrm{L}$. & $9.4 \pm 0.78$ & $0 \pm 0$ & $0 \pm 0$ & $21.7 \pm 1.87$ & $9.2 \pm 0.76$ & $12.7 \pm 1.78$ & $3.7 \pm 0.51$ & $0 \pm 0 ; 2.3 \pm 0.16$ \\
\hline Bignoniaceae & Campsis radicans (L.) Seem. & $0 \pm 0$ & $0 \pm 0$ & $18.6 \pm 0.94$ & $20.5 \pm 2.22$ & $3.3 \pm 0.19$ & $13.5 \pm 1.11$ & $3.7 \pm 0.35$ & $0 \pm 0 ; 2.3 \pm 0.20$ \\
\hline Bignoniaceae & Catalpa duclouxii Dode & $4.4 \pm 0.44$ & $0 \pm 0$ & $10.2 \pm 0.78$ & $22.3 \pm 1.87$ & $0 \pm 0$ & $12.3 \pm 1.88$ & $0 \pm 0$ & $0 \pm 0 ; 2.2 \pm 0.19$ \\
\hline Calycanthaceae & Chimonanthus praecox (L.) Link & $8.7 \pm 0.76$ & $0 \pm 0$ & $8.5 \pm 0.76$ & $23.7 \pm 3.11$ & $0 \pm 0$ & $10.7 \pm 1.49$ & $4.7 \pm 0.45$ & $0 \pm 0 ; 2.2 \pm 0.16$ \\
\hline Celastraceae & Celastrus scandens $\mathrm{L}$. & $0 \pm 0$ & $0 \pm 0$ & $4.6 \pm 0.44$ & $22.6 \pm 2.78$ & $7.2 \pm 0.75$ & $12.6 \pm 1.67$ & $8.4 \pm 0.78$ & $0 \pm 0 ; 2.2 \pm 0.18$ \\
\hline Colchicaceae & Colchicum autumnale L. & $0 \pm 0$ & $0 \pm 0$ & $0 \pm 0$ & $22.7 \pm 2.68$ & $2.8 \pm 0.15$ & $12.7 \pm 1.19$ & $1.6 \pm 0.18$ & $0 \pm 0 ; 2.4 \pm 0.20$ \\
\hline Dennstaedtiaceae & Pteridium aquilinum (L.) Kuhn & $4.2 \pm 0.33$ & $0 \pm 0$ & $11.4 \pm 1.21$ & $21.8 \pm 2.78$ & $3.8 \pm 0.23$ & $12.8 \pm 1.76$ & $3.2 \pm 0.35$ & $0 \pm 0 ; 2.4 \pm 0.21$ \\
\hline Eucommiaceae & Eucommia ulmoides Oliv. & $0 \pm 0$ & $0 \pm 0$ & $0 \pm 0$ & $22.7 \pm 3.21$ & $6.7 \pm 0.24$ & $13.7 \pm 0.87$ & $0 \pm 0$ & $0 \pm 0 ; 2.2 \pm 0.17$ \\
\hline Fabaceae & Genista tinctoria $\mathrm{L}$. & $0 \pm 0$ & $0 \pm 0$ & $12.8 \pm 1.23$ & $23.6 \pm 2.12$ & $3.1 \pm 0.23$ & $13.6 \pm 1.67$ & $1.8 \pm 0.32$ & $0 \pm 0 ; 2.2 \pm 0.16$ \\
\hline Fabaceae & Laburnum anagyroides Medik. & $0 \pm 0$ & $0 \pm 0$ & $3.8 \pm 0.42$ & $21.5 \pm 2.88$ & $0 \pm 0$ & $10.5 \pm 1.78$ & $3.1 \pm 0.32$ & $0 \pm 0 ; 2.4 \pm 0.18$ \\
\hline Fabaceae & Securigera varia (L.) Lassen & $2.1 \pm 0.11$ & $0 \pm 0$ & $3.4 \pm 0.33$ & $20.3 \pm 1.89$ & $0 \pm 0$ & $12.3 \pm 1 . ! 8$ & $0 \pm 0$ & $0 \pm 0 ; 2.2 \pm 0.19$ \\
\hline Fabaceae & $\begin{array}{l}\text { Styphnolobium japonicum } \\
\text { (L.) Schott }\end{array}$ & $0 \pm 0$ & $0 \pm 0$ & $0 \pm 0$ & $22.6 \pm 2.78$ & $0 \pm 0$ & $12.6 \pm 1.78$ & $0 \pm 0$ & $0 \pm 0 ; 2.3 \pm 0.16$ \\
\hline Fabaceae & Wisteria sinensis (Sims) Sweet & $0 \pm 0$ & $0 \pm 0$ & $9.8 \pm 0.31$ & $21.8 \pm 2.76$ & $0 \pm 0$ & $12.8 \pm 1.15$ & $0 \pm 0$ & $0 \pm 0 ; 2.4 \pm 0.21$ \\
\hline Fagaceae & Quercus castaneifolia C. A. Mey. & $0 \pm 0$ & $0 \pm 0$ & $2.9 \pm 0.21$ & $23.8 \pm 4.01$ & $0 \pm 0$ & $10.8 \pm 1.89$ & $3.2 \pm 0.35$ & $0 \pm 0 ; 2.3 \pm 0.19$ \\
\hline Fagaceae & $\begin{array}{l}\text { Q. petraea iberica } \\
\text { (Steven ex M.Bieb.) Krassiln. }\end{array}$ & $16.2 \pm 1.23$ & $0 \pm 0$ & $1.7 \pm 0.13$ & $20.5 \pm 2.78$ & $2.5 \pm 0.14$ & $12.5 \pm 1.87$ & $0 \pm 0$ & $0 \pm 0 ; 2.4 \pm 0.21$ \\
\hline Geraniaceae & Geranium sanguineum $\mathrm{L}$. & $11.3 \pm 0.97$ & $0 \pm 0$ & $10.4 \pm 1.02$ & $21.2 \pm 2.89$ & $5.4 \pm 0.44$ & $9.2 \pm 1.38$ & $0 \pm 0$ & $0 \pm 0 ; 2.4 \pm 0.19$ \\
\hline Ginkgoaceae & Ginkgo biloba L. & $8.4 \pm 0.87$ & $0 \pm 0$ & $1.9 \pm 0.15$ & $22.8 \pm 2.21$ & $2.3 \pm 0.18$ & $11.8 \pm 1.13$ & $1.2 \pm 0.15$ & $0 \pm 0 ; 2.2 \pm 0.16$ \\
\hline Lamiaceae & Callicarpa bodinieri H. Lév. & $10.7 \pm 1.21$ & $0 \pm 0$ & $10.8 \pm 0.99$ & $23.7 \pm 2.56$ & $5.8 \pm 0.31$ & $1.7 \pm 1.31$ & $1.6 \pm 0.16$ & $0 \pm 0 ; 2.3 \pm 0.18$ \\
\hline Lamiaceae & Nepeta racemosa Lam. & $0 \pm 0$ & $0 \pm 0$ & $8.8 \pm 0.91$ & $22.8 \pm 2.77$ & $0 \pm 0$ & $11.8 \pm 0.98$ & $0 \pm 0$ & $0 \pm 0 ; 2.3 \pm 0.20$ \\
\hline Lamiaceae & Salvia officinalis L. & $8.2 \pm 0.87$ & $0 \pm 0$ & $11.2 \pm 1.21$ & $19.7 \pm 2.18$ & $4.4 \pm 0.34$ & $10.7 \pm 1.66$ & $3.4 \pm 0.33$ & $0 \pm 0 ; 2.4 \pm 0.21$ \\
\hline Lamiaceae & Vitex agnus-castus $\mathrm{L}$. & $0 \pm 0$ & $0 \pm 0$ & $3.4 \pm 0.31$ & $23.4 \pm 2.56$ & $10.6 \pm 0.78$ & $11.4 \pm 0.87$ & $0 \pm 0$ & $0 \pm 0 ; 2.4 \pm 0.22$ \\
\hline Lamiaceae & Vitex negundo L. & $0 \pm 0$ & $0 \pm 0$ & $13.4 \pm 1.44$ & $21.5 \pm 1.67$ & $4.2 \pm 0.31$ & $10.1 \pm 0.87$ & $1.3 \pm 0.13$ & $0 \pm 0 ; 2.4 \pm 0.21$ \\
\hline Magnoliaceae & Liriodendron tulipifera $\mathrm{L}$. & $10.3 \pm 1.13$ & $0 \pm 0$ & $11.8 \pm 1.13$ & $21.7 \pm 4.02$ & $3.6 \pm 0.24$ & $10.7 \pm 0.87$ & $9.4 \pm 1.23$ & $0 \pm 0 ; 2.2 \pm 0.16$ \\
\hline Magnoliaceae & Magnolia kobus DC. & $10.8 \pm 1.21$ & $0 \pm 0$ & $1.2 \pm 0.19$ & $20.3 \pm 2.21$ & $8.6 \pm 0.56$ & $12.3 \pm 1.89$ & $4.4 \pm 0.46$ & $0 \pm 0 ; 2.4 \pm 0.21$ \\
\hline Moraceae & $\begin{array}{l}\text { Machura pomifera (Raf.) } \\
\text { C. K. Schneid. }\end{array}$ & $10.5 \pm 0.88$ & $0 \pm 0$ & $10.8 \pm 0.87$ & $20.7 \pm 3.29$ & $10.9 \pm 1.12$ & $9.7 \pm 1.56$ & $0 \pm 0$ & $0 \pm 0 ; 2.4 \pm 0.18$ \\
\hline Ranunculaceae & Clematis flammula L. & $10.3 \pm 1.11$ & $0 \pm 0$ & $4.5 \pm 0.41$ & $23.6 \pm 2.31$ & $10.5 \pm 1.31$ & $11.6 \pm 1.19$ & $1.2 \pm 0.12$ & $0 \pm 0 ; 2.4 \pm 0.19$ \\
\hline Rosaceae & Prunus dulcis (Mill.) D. A. Webb & $2.3 \pm 0.23$ & $0 \pm 0$ & $10.6 \pm 1.22$ & $20.1 \pm 2.78$ & $2.5 \pm 0.18$ & $12.1 \pm 0.87$ & $1.3 \pm 0.15$ & $0 \pm 0 ; 2.4 \pm 0.22$ \\
\hline Rosaceae & Prunus laurocerasus $\mathrm{L}$. & $0 \pm 0$ & $0 \pm 0$ & $16.5 \pm 1.34$ & $20.8 \pm 2.33$ & $1.3 \pm 0.08$ & $10.8 \pm 1.32$ & $0 \pm 0$ & $0 \pm 0 ; 2.4 \pm 0.21$ \\
\hline Rutaceae & Dictammus alba $\mathrm{L}$. & $2.2 \pm 0.19$ & $0 \pm 0$ & $2.3 \pm 0.21$ & $22.8 \pm 2.44$ & $2.7 \pm 0.16$ & $13.8 \pm 1.78$ & $1.7 \pm 0.18$ & $0 \pm 0 ; 2.3 \pm 0.18$ \\
\hline Rutaceae & Phellodendron amurense Rupr. & $9.7 \pm 0.94$ & $0 \pm 0$ & $12.9 \pm 1.42$ & $22.6 \pm 2.65$ & $10.2 \pm 0.76$ & $12.6 \pm 1.32$ & $8.7 \pm 0.67$ & $0 \pm 0 ; 2.3 \pm 0.17$ \\
\hline Rutaceae & Ptelea trifoliata $\mathrm{L}$. & $17.5 \pm 1.56$ & $0 \pm 0$ & $10.3 \pm 0.88$ & $19.7 \pm 2.42$ & $1.7 \pm 0.09$ & $11.7 \pm 1.32$ & $3.3 \pm 0.36$ & $0 \pm 0 ; 2.4 \pm 0.18$ \\
\hline Sapindaceae & Koelrenteria paniculata Laxm. & $13.3 \pm 1.23$ & $0 \pm 0$ & $2.4 \pm 0.21$ & $21.7 \pm 3.27$ & $2.3 \pm 0.13$ & $12.7 \pm 1.44$ & $6.4 \pm 0.42$ & $0 \pm 0 ; 2.5 \pm 0.20$ \\
\hline Simaroubaceae & Ailanthus altissima (Mill.) Swingle & $2.2 \pm 0.31$ & $0 \pm 0$ & $2.3 \pm 0.21$ & $19.7 \pm 1.97$ & $0 \pm 0$ & $11.7 \pm 1.69$ & $3.6 \pm 0.47$ & $0 \pm 0 ; 2.3 \pm 0.18$ \\
\hline Tamaricaceae & Tamarix elongata Ledeb. & $11.4 \pm 1.15$ & $0 \pm 0$ & $11.4 \pm 0.89$ & $20.4 \pm 2.34$ & $0 \pm 0$ & $10.4 \pm 1.65$ & $0 \pm 0$ & $0 \pm 0 ; 2.4 \pm 0.21$ \\
\hline Vitaceae & $\begin{array}{l}\text { Parthenocissus tricuspidata } \\
\text { (Siebold \& Zucc.) Planch. }\end{array}$ & $10.6 \pm 0.88$ & $0 \pm 0$ & $4.3 \pm 0.35$ & $19.9 \pm 2.13$ & $5.3 \pm 0.34$ & $14.9 \pm 1.23$ & $10.7 \pm 1.24$ & $0 \pm 0 ; 2.2 \pm 0.17$ \\
\hline
\end{tabular}

Note: $*$ - disks with $15.0 \mu \mathrm{g}$ of azithromycin were used for all bacteria as positive control; ${ }^{* *}$ - discs with $15.0 \mu \mathrm{g}$ amphotericin were used for all bacteria as positive control for Candida albicans.

Fungicidal activity of essential oil from Chimonanthus praecox L. was observed towards eight phytopathogenic fungi, the inhibiting power measuring 8-32 $\mu \mathrm{g} / \mathrm{mL}$ (Gui \& Qin, 2014).

Adami \& Naderi (2015) think that the most important compound in the plant Colchicum autumnale L. is colchicine alkaloid, though its antimicrobial activity is studied poorly.

Ethanolic and petroleum extracts of Pteridium aquilinum (L.) exhibit antibacterial properties (Kardong \& Saikia, 2013) against four species of tested bacteria (B. subtilis, S. aureus, $P$. vulgaris and $E$. coli), producing inhibition zones ranging 16-20 mm. Bacteria of $P$. aeruginosa were resistant to extracts of this species (Kardong \& Saikia, 2013). However, extracts prepared in methanol, chloroform and distilled water showed no inhibiting activity against all the tested organisms. The observed difference in antibacterial activity while using various methods of extraction may be explained by incomplete transition of active substances into solution in the temperature of environment and loss of active components during boiling (Kardong \& Saikia, 2013).

Antimicrobial peptides arrest the growth of bacteria, fungi, plant pathogens and even viruses. They have a powerful pharmaceutical effect. Bark of Eucommia ulmoides Oliv. is used in traditional Chinese medicine. Peptide present in E. ulmoides had in vitro inhibiting effect (Liu \& Han, 2007) on Candida albicans (MIC $=156 \mu \mathrm{g} / \mathrm{mL}$ ). Liu \& Han (2007) consider that this plant can be used as a new antibiotic of plant origin for pre- vention of candidosis. Screening of bioactive secondary metabolites demonstrated that roots of $E$. ulmoides contain 7 compounds, one of them being gliotoxin. Its activity was close to the activity of gentamicin antibiotic, and stronger that the activity of nystatin antifungal preparation (Zhang \& An, 2019)

Extracts of Genista tinctoria contributed to proliferation of probiotic strains and increased the number of bacterial colonies of Bifidobacterium animalis subsp. lactis, B. longum and Lactobacillus casei (Skenderidis \& Giavasis, 2019). Prebiotic effect correlates with the concentration of polysaccharides and polyphenols of $G$. tinctoria, the content of which can increase the stress-tolerance of $B$. lactis and $B$. longum in a modelled gastrointestinal environment. Skenderidis \& Giavasis (2019) consider that encapsulated extracts from $G$. tinctoria could be used as prebiotic supplements for food products for stimulation of growth and increase in vitality of probiotic strains of Bifidobacterium and Lactobacillus.

Oak (Quercus petraea subsp. iberica (Steven ex M. Bieb.) Krassiln) has many medical properties. Tumen \& Sekeroglu (2018) report antiinflammatory, wound-healing, anthelmintic and antioxidant effects of this plant. Extract of leaves of $Q$. petraea had antimicrobial activity against Listeria monocytogenes. Inhibiting effect of $Q$. petraea was more strongly expressed at low temperature $\left(4^{\circ} \mathrm{C}\right)$, and addition of EDTA (ethylenediaminetetraacetic acid) increased its antimicrobial activity (Xie \& Johnson, 2003). 
Quercus castaneifolia is being clinically tested regarding the treatment of patients suffering from intestinal diseases caused by E. coli, $S$. typhimurium, Shigella dysenteriae, Y. enterocolitica (Bahador \& Baserisalehi, 2011).

Oil extract from Geranium sanguineum L. exhibited antibacterial effects against one standard strain of S. aureus ATCC 433000 and seventy clinical strains of $S$. aureus, including strains with multi-drug resistance (Bigos \& Sienkiewicz, 2012). Wafa \& Ouarda (2017) report high antimicrobial activities of methanol extracts of $G$. sanguineum against $S$. aureus ATCC 25923, E. coli ATCC 25922, P. aeruginosa ATCC 27853, B. subtilis ATCC 6633 and C. albicans ATCC1024, while having moderate anti-inflammatory effect.

Iridodial $\beta$-monoenol acetate and actinidine obtained from extract of Nepeta racemosa Spreng. demonstrated high activity against Penicillium citrinum and Aspergillus spp. and moderate zone of inhibition of Bacillus anthracis and Streptococcus pyrogenes (Saxena \& Mathela, 1996; Mathela \& Joshi, 2008).

Biofilms containing solid dispersion of extract from Salvia officinalis L. had high antibacterial activity towards food pathogens $S$. aureus and E. coli (Salević \& Lagaron 2019; Wali \& Alam, 2019).

Habbab \& Aboul-Enein (2016) studied the chemical composition and biological activity of essential oils from dry leaves, flowers and seeds of Vitex agnus-castus L., obtained using hydrodistillation. Antifungal and antibacterial activities of essential oil was tested against three strains of fungi and eight strains of bacteria. The main constituents of the oil were 1,8-cineol (17.2\%), caryophyllene (12.9\%) and terpinen-4-ol (10.2\%), whereas the dominating compounds in the oil of seeds were 1,8-cineol (14.9\%), cedrelanol (13.9\%) and 7a-isopropenile-4,5-dimethyloctahydroindene-4-carbonic acid (13.9\%). Oil from leaves also contained 1,8-cineol (18.3\%). Their compositions were compared to oils of plants from Europe, America and Asia. Essential oils of seeds and leaves exerted antibacterial impact against $K$. pneumoniae, E. coli and $P$. aeruginosa. Essential oil from leaves and flowers was highly active against fungi Penicillium expansum and Aspergillus flavus.

Plant extract of Vitex negundo L. was the most effective (Padder \& Ganaie, 2015) both against Streptococcus mutans (MIC - minimum inhibitory concentration $=0.37 \mu \mathrm{g} / \mathrm{mL}$ ) and $P$. aeruginosa $(\mathrm{MIC}=$ $0.75 \mu \mathrm{g} / \mathrm{mL}$ ). Deogade et al. (2016) determined antibacterial activity of ethanol extract from leaves of $V$. negundo towards bacteria Escherichia coli, Staphylococcus aureus and Klebsiella pneumoniae, producing maximum inhibition zone of $S$. aureus $(15 \mathrm{~mm}$ at the concentration of 80 $100 \mathrm{mg} / \mathrm{mL}$ ) and minimum ones of E. coli and K. pneumoniae (12 and $11 \mathrm{~mm}$ at the concentration of $100 \mathrm{mg} / \mathrm{mL}$, respectively). The notable inhibiting activity of extract of this plant was due to its high content of phenols and flavonoids (Prashith \& Raghavendra, 2014). Extracts and secondary metabolites of $V$. negundo, especially from the roots and leaves, have useful pharmacological properties: anti-inflammatory, anti-tumour, antioxidant and antimicrobial (Tan et al., 2017; Khan et al., 2019). Use of $V$. negundo may be promising for treatment of skin infections caused by Staphylococcus aureus (Triveni \& Gaddad, 2016). Synthesis of nanoparticles of silver through self-restoration of silver nitrate by extracts of leaves of $V$. negundo is one of the new methods applied in the development of technologies for creation of nanoparticles (Bhavani \& Geetha, 2013). Silver nanoparticles $(56 \mathrm{~nm})$ exhibited antimicrobial activity against $E$. coli and $K$. pneumonia. Essential oil from seeds of $V$. negundo (Ai, 2014) had significant antifungal impact on Candida albicans (MIC = $4.0 \mu \mathrm{g} / \mathrm{mL}$ ). Moreover, this extract had hepatoprotectory properties, which could be associated with its antioxidant activity, and also protective effect against heightened level of lipids (Sharma \& Suri, 2016).

Alcohol extracts from the pith of Liriodendron tulipifera L. showed antimicrobial activity towards S. aureus, Mycobacterium smegmatis, Candida albicans and Aspergillus niger. Hufford \& Robertson (1975) attribute it to alkaloid fraction of dehydro glaucine and liriodenine as active components. Mechanisms of antimicrobial activity of extract from Magnolia kobus DC. on S. aureus were studied using light microscopy, transmission electronic microscopy and scanning electron microscopy. After $48 \mathrm{~h}$ of exposure to the extract, many cells of $S$. aureus completely decomposed (Hu \& Ge, 2011). Methylene chloride extract from fruits of Maclura pomifera (Raf.) Schneid exerted strong in vitro antimicrobial and
anti-Leishmania activities. Fractioning of this extract based on the activity led to production of isoflavons (osajin and pomiferin) as active compounds which demonstrated high activity against Cryptococcus neoformans, Staphylococcus aureus and Leishmania donovani (Dharmaratne \& Nanayakkara, 2013). Lectin from M. pomifera in a specific way agglutinated bacterial suspensions of various strains of highly-pathogenic bacteria of Salmonella genus (Allen, 1985).

Methanol extracts of leaves and bark of Clematis flammula L. exhibited a broad spectrum of antibacterial activity due to fraction of ethylacetate (Khan \& Omoloso, 2001). Non-filtered extracts of young shoots of the closely related plant Clematis vitalba $\mathrm{L}$. were highly-active against pathogenic yeasts and yeast-like microorganisms ( $\mathrm{MIC}=1.4-12.3$ $\mu \mathrm{g} / \mathrm{mL})$. After fractioning with petroleum alcohol, ethylacetate and methanol, antifungal activity was observed only in methanol fractions (Buzzini \& Pieroni, 2003).

Thebo (2014) surveyed the extract of the shell of Prunus dulcis (Mill.) in its biomedical aspects: antifungal activity of extract of almond shell was observed against the clinically isolated pathogenic fungus Tinea capitis using the strip method. The antioxidant potential of non-filtered extract of the coating of the fruits was also assessed using DPPH $(2,2-$ diphenyl-1-picrylhydrazyl) and the system of scaveranging of radicals. The total antioxidant activity ranged 94.4-95.5\%; total content of phenols accounted for $4.46 \mathrm{mg} / \mathrm{g}$ in the extract of almond shell. This had a great therapeutic potential after 20 days of therapy against $T$. capitis-caused infection of the skin on the head. The survey has proven the clinical efficiency of Prunus dulcis for treating dermatological diseases.

Antimicrobial activity of essential oil from Dictammus dasycarpus Turcz. was tested against nine microorganisms using methods of disk diffusions and broth microdilutions. The essential oil displayed bactericidal activity towards S. aureus ATCC 25923 and methicillin-resistant strain of S. aureus (Lei \& Liao, 2007).

Extracts from bark of Phellodendron amurense Rupr. were tested for antioxidant, antimicrobial and antiviral activities (against virus of herpes simplex of 1 type - HSV-1). Ethanol extract from the bark of this plant, compared with the aqueous extract, was found to contain more phenols and flavonoids. The ethanol extract was much more active against bacteria than the aqueous extract (Wang \& Zhang, 2009). Han \& Meng (2015) surveyed essential oil from $P$. amurense extracted from fruits collected in the natural growing environment. This oil contained myrcene $(51.7 \%)$, 2-methyl-6-methylene-octa-3,7-dien-2-ol (7.4\%), 1,2-benzenedioic acidbi-(2-methylpropyl)-complex ether (7.2\%), 2-methyl -6-methylene-octa1,7-dien-3-ol (7.1\%) and $\alpha$-phellandrene (5.2\%). Essential oil from $P$. amurense demonstrated antioxidant $\left(\mathrm{IC}_{50}-2.32 \mu \mathrm{g} / \mathrm{mL}\right)$ and broadrange fumigant activity and notable antimicrobial effect against all the tested strains of microorganisms ( $\mathrm{MIC}=0.12-1.36 \mu \mathrm{g} / \mathrm{mL}$ ). Han \& Meng (2015) assume that the essential oil can be used as an antioxidant and antimicrobial agent.

Bioanalysis-based fractioning of ethanol extract of Koelreuteria paniculata Laxm. of the Sapindaceae family, which grows in Egypt, led to isolation of 11 compounds: methyl-myo-inositol, loliloide, gallic acid, methyl gallate, ethyl gallate, monoglyceride of palmitic acid, 5-meth-oxyluteolin, kaempferol-7-rhamnoside, kaempferol-3- rhamnoside, $\beta$-sitosterol and $\beta$-sitosterol-glucoside. Methyl gallate and ethyl gallate showed identical anti-malaria activity against chloroquine-susceptible and insusceptible forms of Plasmodium falciparum. Ethyl gallate was also acive against $E$. coli bacteria (Mostafa \& Ross, 2015).

Methanol extracts from leaves of Ailanthus altissima (Mill.) Swingle and their hydrodistilled residuals have antioxidant, phytotoxic and antibacterial activities against Gram-positive bacterial strains (Albouchi \& Hosni, 2013).

Methanol extracts of Parthenocissus tricuspidata (Siebold \& Zucc.) Planch. demonstrated in vitro anti-malaria activity against Plasmodium falciparum, and also a schizonticidal activity towards $P$. berghei in blood of mice in the conditions of use of the doses causing no noticeable toxicity: these extracts elevated the share of oxidized hemoglobin in erythrocytes and inhibited synthesis of protein (Park \& Moon, 2008).

The antimicrobial impact of the remaining species of plants is covered much less thoroughly in the literature. Thus, according to our results, ethanol extracts inhibit growth of colonies of many species of microorga- 
nisms of the Yersiniaceae, Enterobacteriaceae, Morganellaceae, Enterococcaceae, Listeriaceae, Pseudomonadaceae, Campylobacteraceae, Corynebacteriaceae, Nocardiaceae families and fungi of the Saccharomycetacea family. A somewhat disturbing find was that the strains of $P$. mirabilis, $K$. pneumoniae, $S$. marcescens, L. monocytogenes and $C$. jejuni, which we studied, were absolutely resistant to azithromycin (growth inhibition zone equaled $0.0 \mathrm{~mm}$ ), and that $C$. albicans showed a low susceptibility to amphotericin (growth inhibition zone was $2.4 \mathrm{~mm}$ ).

\section{Conclusion}

For the first time a study on the complex inhibitory action of 38 species of plants against 15 bacterial strains and one strain of fungus has been undertaken. Extracts of leaves and shoots of plants of Fabaceae (Styphnolobium japonicum, Securigera varia), Rutaceae (Dictamnus albus), Lamiaceae (Nepeta racemosa), Eucommiaceae (Eucommia ulmoides), Rosaceae (Prunus dulcis), Bignoniaceae (Campsis radicans), Simaroubaceae (Ailanthus altissima) and Fagaceae (Quercus petraea) had no notable effect on multi-ressitant strains of $E$. coli, P. mirabilis, $S$. marcescens, L. ivanovi, L. monocytogenes, $P$. aeruginosa, C. jejuni and $C$. albicans. We determined intense inhibiting effect of ethanol extracts from Maclura pomifera, Ginkgo biloba against 8, Genista tinctoria, Phellodendron amurense, Berberis vulgaris - 7, Vitex negundo, Koelreuteria paniculata, Magnolia kobus, Liriodendron tulipifera, Clematis flammula - 6, Wisteria sinensis, Chimonanthus praecox, Colchicum autumnale - 5, Vitex agnus-castus, Salvia officinalis, Prunus laurocerasus, Geranium sanguineum, Tamarix elongata, Catalpa duclouxii, Parthenocissus tricuspidata, Quercus castaneifolia - 4, Artemisia absinthium, Ptelea trifoliata, Polygonatum multiflorum, Kalicopa bodimerium, Aristolochia manshuriensis, Celastrus scandens and Pteridium aquilinum - 3 of 16 surveyed multi-drug-resitant strains of bacteria and fungi. We consider it possible to recommend ethanol extracts from M. pomifera, G. biloba, G. tinctoria, P. amurense, B. vulgaris, $V$. negundo, K. paniculata, M. kobus, L. tulipifera, C. flammula, W. sinensis, $C$. praecox and $C$. autumnale or the individual compounds these plants contain for further research on combating poly-resistant strains of the abovementioned microorganisms.

\section{References}

Adami, R., Nayeri, H., \& Naderi, G. (2015). Anti-diabetic mechanism of colchicum speciosum hydroalcoholic extract in vitro. International Journal of Biosciences, 6(5), 152-157.

Ai, H.-W. (2014). Antifungal properties and chemical analysis of essential oil from Vitex negundo seeds. British Journal of Pharmaceutical Research, 4(5), 541-548.

Akpulat, H. A., Akpulat, S., Yildirim, E. S., \& Enginoğlu, H. R. (2019). Prunus laurocerasus (Rosaceae) plant extract with harmful herbs and agricultural frost. Turkish Journal of Biodiversity, 2(1), 18-23.

Albouchi, F., Hassen, I., Casabianca, H., \& Hosni, K. (2013). Phytochemicals, antioxidant, antimicrobial and phytotoxic activities of Ailanthus altissima (Mill.) Swingle leaves. South African Journal of Botany, 87, 164-174.

Al-Ghamdi, A. A. M. (2020). Ecological and biochemical studies on Artemisia absinthium in Al-Baha city, Saudi Arabia. Pakistan Journal of Botany, 52(4), 14.

Allen, P. Z. (1985). Interaction of Salmonella telaviv with Machra pomifera lectin. Infection and Immunity, 47(1), 90-93.

Anzabi, Y. (2018). Biosynthesis of $\mathrm{ZnO}$ nanoparticles using barberry (Berberis vulgaris) extract and assessment of their physico-chemical properties and antibacterial activities. Green Processing and Synthesis, 7(2), 114-121.

Bahador, N., \& Baserisalehi, M. (2011). The effect of Quercus castaneifolia extract on pathogenic enteric bacteria. Anaerobe, 17(6), 358-360.

Bährle-Rapp, M. (2007). Polygonum multiflorum extract. In: Bahrle-Rapp, M. Springer Lexikon Kosmetik Und Körperpflege. Springer-Verlag, Berlin, Heidelberg. Pp. $439-439$.

Behbahani, M., Shanehsazzadeh, M., Shokoohinia, Y., \& Soltani, M. (2013). Evaluation of anti-herpetic activity of methanol seed extract and fractions of Securigera securidaca in vitro. Journal of Antivirals and Antiretrovirals, 2013, 5,4 .

Bhavani, G., Muthuselvam, P., \& Geetha, S. (2013). Synthesis of silver nanoparticles using the leaf extract of Vitex negundo and its antibacterial effect. Advanced Materials Research, 678, 301-305.

Bigos, M., Wasiela, M., Kalemba, D., \& Sienkiewicz, M. (2012). Antimicrobial activity of Geranium oil against clinical strains of Staphylococcus aureus. Molecules, 17(9), 10276-10291.
Boyko, A. A., \& Brygadyrenko, V. V. (2016a). Influence of water infusion of medicinal plants on larvae of Strongyloides papillosus (Nematoda, Strongyloididae). Visnyk of Dnipropetrovsk University, Biology, Ecology, 24(2), 519-525.

Boyko, O. O., Zazharska, N. M., \& Brygadyrenko, V. V. (2016b). The influence of the extent of infestation by helminths upon changes in body weight of sheep in Ukraine. Visnyk of Dnipropetrovsk University, Biology, Ecology, 24(1), 3-7.

Buzzini, P., \& Pieroni, A. (2003). Antimicrobial activity of extracts of Clematis vitalba towards pathogenic yeast and yeast-like microorganisms. Fitoterapia, 74(4), 397-400.

Compton, J. A. (2015). 815. Wisteria sinensis on the slow boat from China: The journey of Wisteria to England. Curtis's Botanical Magazine, 32, 248-293.

Deogade, M. S., Pandya, T., Prasad, K. S., Kale, K., \& Tankhiwale, N. (2016). Antimicrobial activity of Vitex negundo Linn. (Nirgundi) leaves extract. Journal of Research in Traditional Medicine, 2(4), 99-102.

Dharmaratne, H., Jacob, M., Tekwani, B., \& Nanayakkara, N. (2013). Antimicrobial and antileishmanial compounds from Maclura pomifera fruits. Planta Medica, 79, PF1.

Geraldes, M., Fonseca, J. P., Neto, C., \& Costa, J. C. (2019). New genetic data on Genista anglica L. versus Genista ancistrocarpa Spach (Fabaceae, Fabales) in the Iberian Península and Morocco. Phylogeographic clues. Acta Botanica Malacitana, 39, 45-54.

Gui, R.-Y., Liang, W.-W., Yang, S.-X., Llu, L., \& Qin, J.-C. (2014). Chemical composition, antifungal activity and toxicity of essential oils from the leaves of Chimonanthus praecox located at two different geographical origin. Asian Journal of Chemistry, 26(14), 4445-4448.

Habbab, A., Sekkoum, K., Belboukhari, N., Cheriti, A., \& Aboul-Enein, H. Y. (2016). Essential oil chemical composition of Vitex agnus-castus L. from Southern-West Algeria and its antimicrobial activity. Current Bioactive Compounds, 12(1), 51-60.

Han, Y., Xu, L., Wang, Q., Huang, Y., \& Meng, W. (2015). Chemical composition, antioxidant and antimicrobial activity of the essential oil of Phellodendron amurense (Rupr.) from China. Asian Journal of Chemistry, 27(3), 841-844.

Hu, Y., Qiao, J., Zhang, X., \& Ge, C. (2011). Antimicrobial effect of Magnolia officinalis extract against Staphylococcus aureus. Journal of the Science of Food and Agriculture, 91(6), 1050-1056.

Hufford, C. D., Funderburk, M. J., Morgan, J. M., \& Robertson, L. W. (1975). Two antimicrobial alkaloids from heartwood of Liriodendron tulipifera L. Joumal of Pharmaceutical Sciences, 64(5), 789-792.

Islam, M., Jannat, T., Kuddus, M. R., Rashid, M. A., \& Haque, M. R. (2019). In vitro and in vivo evaluation of pharmacological potentials of Campsis radicans $\mathrm{L}$. Clinical Phytoscience, 5, 42.

Kardong, D., Upadhyaya, S., \& Saikia, L. R. (2013). Screening of phytochemicals, antioxidant and antibacterial activity of crude extract of Pteridium aquilinum Kuhn. Journal of Pharmacy Research, 6(1), 179-182.

Kavitha, T., Alagusaranya, A., \& Nelson, R. (2016). Antimicrobial activity of chloroform extract of Aristolochia bracteata Retz. and analysis of bioactive compounds. International Journal of Current Microbiology and Applied Sciences, 5(3), 559-565.

Khan, M. F., Arora, P., \& Dhobi, M. (2019). A prospective review on phyto-pharmacological aspects of Vitex negundo Linn. Current Traditional Medicine, 2019, in print.

Khan, M., Kihara, M., \& Omoloso, A. (2001). Antimicrobial activity of Clematis papuasica and Nauclea obversifolia. Fitoterapia, 72(5), 575-578.

Kumar, V., Singh, S., Singh, A., Dixit, A. K., Shrivastava, B., Kondalkar, S. A., Singh, J., Singh, R., Sidhu, G. K., Singh, R. P., Subhose, V., \& Prakash, O. (2018). Determination of phytochemical, antioxidant, antimicrobial, and protein binding qualities of hydroethanolic extract of Celastrus paniculatus. Journal of Applied Biology and Biotechnology, 6(6), 11-17.

Lei, J., Yu, J., Yu, H., \& Liao, Z. (2007). Composition, cytotoxicity and antimicrobial activity of essential oil from Dictamnus dasycarpus. Food Chemistry, 107(3), 1205-1209.

Lim, T. K. (2013). Styphnolobium japonicum. In: Lim, T. K. (Ed.). Edible medicinal and non-medicinal plants. Springer, Dordrecht. Pp. 906-924.

Liu, S., Zhao, D., \& Han, Y. (2007). An antifungal peptide from the bark of Eucommia ulmoides Oliv. effective against Candida albicans in vitro. International Symposium on Eucommia ulmoides, 1(1), 78-81.

Lopes, A., Pereira, C., \& Almeida, A. (2018). Sequential combined effect of phages and antibiotics on the inactivation of Escherichia coli. Microorganisms, 6(4), 125.

Ma, Z., \& Su, Z. (2015). Lectotypification of Callicarpa nudiflora (Lamiaceae). Phytotaxa, 224(1), 100.

Mathela, C. S., \& Joshi, N. (2008). Antimicrobial activity of Nepeta isolates. Natural Product Communications, 3(6), 625.

Mostafa, A., El-Hela, A., Mohammad, A., Cutler, S., \& Ross, S. (2015). Antimalarial compounds isolated from Koelreuteria paniculata growing in Egypt. Planta Medica, 81, 5 .

Obistioiu, D., Cristina, R. T., Schmerold, I., Chizzola, R., Stolze, K., Nichita, I., \& Chiurciu, V. (2014). Chemical characterization by GC-MS and in vitro activity against Candida albicans of volatile fractions prepared from Artemisia 
dracunculus, Artemisia abrotanum, Artemisia absinthium and Artemisia vulgaris. Chemistry Central Journal, 8, 6 .

Özgen, M., Saraçoğlu, O., \& Geçer, E. N. (2012). Antioxidant capacity and chemical properties of selected barberry (Berberis vulgaris L.) fruits. Horticulture, Environment, and Biotechnology, 53(6), 447-451.

Padder, B., Yasmeen, S., \& Ganaie, M. (2015). Antibiotic/antibacterial activity of Vitex negundo, Duranta repens, Acorus calamus and Piper nigrum. British Biotechnology Journal, 6(1), 16-22.

Palchykov, V. A., Zazharskyi, V. V., Brygadyrenko, V. V., Davydenko, P. O., Kulishenko, O. M., \& Borovik, I. V. (2020). Chemical composition and antibacterial effect of ethanolic extract of Buxus sempervirens on cryogenic strains of microorganisms in vitro. Chemical Data Collections, 25, 100323.

Palchykov, V. A., Zazharskyi, V. V., Brygadyrenko, V. V., Davydenko, P. O., Kulishenko, O. M., Borovik, I. V., Chumak, V., Kryvaya, A., \& Boyko, O. O. (2019). Bactericidal, protistocidal, nematodicidal properties and chemical composition of ethanol extract of Punica granatum peel. Biosystems Diversity, 27(3), 300-306.

Park, W.-H., Lee, S.-J., \& Moon, H.-I. (2008). Antimalarial activity of a new stilbene glycoside from Parthenocissus tricuspidata in mice. Antimicrobial Agents and Chemotherapy, 52(9), 3451-3453.

Prashith, K., Vivek, M., Kambar, Y., Manasa, M., \& Raghavendra, H. (2014). Comparative study on antimicrobial activity of Vitex negundo var. negundo and Vitex negundo var. purpurascens. Science, Technology and Arts Research Journal, 3(1), 126.

Rivers, M. C. (2016). Laburnum anagyroides. In: IUCN Red List of Threatened Species. P. 43.

Saidana, D., Mahjoub, M. A., Boussaada, O., Chriaa, J., Chéraif, I., Daami, M., Mighri, Z., \& Helal, A. N. (2008). Chemical composition and antimicrobial activity of volatile compounds of Tamarix boveana (Tamaricaceae). Microbiological Research, 163(4), 445-455.

Salević, A., Prieto, C., Cabedo, L., Nedović, V., \& Lagaron, J. (2019). Physicochemical, antioxidant and antimicrobial properties of electrospun poly( $\varepsilon$-caprolactone) films containing a solid dispersion of sage (Salvia officinalis L.) extract. Nanomaterials, 9(2), 270.

Saxena, J., \& Mathela, C. S. (1996). Antifungal activity of new compounds from Nepeta leucophylla and Nepeta clarkei. Applied and Environmental Microbiology, 62(2), 702-704.

Sharma, N., \& Suri, J. (2016). Protective effect of a standardized fraction from Vitex negundo Linn. against acetaminophen and galactosamine induced hepatotoxicity in rodents. Biochemistry and Analytical Biochemistry, 5, 2.

Skenderidis, P., Mitsagga, C., Lampakis, D., Petrotos, K., \& Giavasis, I. (2019). The effect of encapsulated powder of goji berry (Lycium barbarum) on growth and survival of probiotic bacteria. Microorganisms, 8(1), 57.
Steinberg, K. M., Satyal, P., \& Setzer, W. N. (2017). Bark essential oils of Zanthoxylum clava-herculis and Ptelea trifoliata: Enantiomeric distribution of monoterpenoids. Natural Product Communications, 12(6), 632.

Tan, L. S., Mousavi, L., \& Salleh, R. M. (2017). Development and characterisation of Vitex negundo Linn. noodles. Food Research, 2(1), 68-75.

Thebo, N. (2014). Clinical study of the Prumus dulcis (Almond) shell extract on Tinea capitis infection. Natural Products Chemistry and Research, 2, 3.

Triveni, A. G., Mendem, S. K., Shivannavar, C. T., \& Gaddad, S. M. (2016). Antibacterial activity of Vitex negundo leave extract against methicillin resistant Staphylococcus aureus (MRSA). International Journal of Pharmacy and Biological Sciences, 6(3), 55-59.

Tumen, I., Eyuboglu, S., Kurtca, M., \& Sekeroglu, N. (2018). Chemical compounds of oak (Quercus petraea (Matt.) Liebl.) species with different medicinal properties in Eastem Black Sea Region. Annals of Phytomedicine, 7(1), 140-146.

Wafa, N., Sofiane, G., \& Ouarda, D. (2017). Antioxidant, antimicrobial and anti-inflammatory activities valorisation of methanol extract of two Geranium species growth in Setif Algeria. International Journal of Pharma Research and Health Sciences, 5(3), 1698-1702.

Wali, A. F., Hamad, E. A., Khazandar, A. A., Al-Azzawi, A. M., Sarheed, O. A., Menezes, G. A., \& Alam, A. (2019). Antimicrobial and in vitro antioxidant activity of Salvia officinalis L. against various re-emergent multidrug resistance microbial pathogens. Annals of Phytomedicine, 8(2), 115-120.

Wang, W., Zu, Y., Fu, Y., Reichling, J., Suschke, U., Nokemper, S., \& Zhang, Y. (2009). In vitro antioxidant, antimicrobial and anti-Herpes simplex virus type 1 activity of Phellodendron amurense Rupr. from China. The American Joumal of Chinese Medicine, 37(1), 195-203.

Xie, L., Hettiarachchy, N. S., Jane, M. E., \& Johnson, M. G. (2003). Antimicrobial activity of Ginkgo biloba leaf extract on Listeria monocytogenes. Journal of Food Science, 68(1), 268-270.

Zazharskyi, V. V., Davydenko, P. O., Kulishenko, O. M., Borovik, I. V., \& Brygadyrenko, V. V. (2019a). Antimicrobial activity of 50 plant extracts. Biosystems Diversity, 27(2), 163-169.

Zazharskyi, V., Davydenko, P., Kulishenko, O., Borovik, I., Brygadyrenko, V., \& Zazharska, N. (2019b). Antibacterial activity of herbal infusions against Staphylococcus aureus, Staphylococcus epidermidis and Pseudomonas aeruginosa in vitro. Magyar Állatorvosok Lapja, 141, 693-704.

Zazharskyi, V., Parchenko, M., Fotina, T., Davydenko, P., Kulishenko, O., Zazharskaya, N., \& Borovik, I. (2019c). Synthesis, structure, physicochemical properties and antibacterial activity of 1,2,4-triazoles-3-thiols and furan derivatives. Voprosy Khimii i Khimicheskoi Tekhnologii, 6, 74-82.

Zhang, H., \& An, Z. (2019). Gliotoxin analogues from endophytic Penicillium sp. of Eucommia ulmoides and their antimicrobial activity. Chemistry of Natural Compounds, 55(4), 793-795.

Zhang, X., Lu, Y., Xu, J., \& Liu, Z. (2018). Leachates of medicinal herbs inhibit the decomposition rate of Catalpa fargesii Bur. litter. Écoscience, 25(2), 179-188. 